\title{
Theoretical Modeling for the STEREO Mission
}

\author{
Markus J. Aschwanden - L. F. Burlaga - M. L. Kaiser • \\ C. K. Ng • D. V. Reames · M. J. Reiner • T. I. Gombosi • \\ N. Lugaz • W. Manchester IV • I. I. Roussev • \\ T. H. Zurbuchen - C. J. Farrugia - A. B. Galvin • \\ M. A. Lee · J. A. Linker · Z. Mikić · P. Riley • \\ D. Alexander $\cdot$ A. W. Sandman · J. W. Cook • \\ R. A. Howard · D. Odstrčil · V. J. Pizzo · J. Kóta • \\ P. C. Liewer · J. G. Luhmann · B. Inhester • \\ R. W. Schwenn - S. K. Solanki · V. M. Vasyliunas • \\ T. Wiegelmann - L. Blush · P. Bochsler · I. H. Cairns • \\ P. A. Robinson • V. Bothmer · K. Kecskemety •

\section{A. Llebaria - M. Maksimovic • M. Scholer •} \\ R. F. Wimmer-Schweingruber
}

Received: 9 November 2005 / Accepted: 7 August 2006 / Published online: 1 November 2006 (C) Springer Science + Business Media B.V. 2007

\begin{abstract}
We summarize the theory and modeling efforts for the STEREO mission, which will be used to interpret the data of both the remote-sensing (SECCHI, SWAVES) and in-situ
\end{abstract}

\author{
M. J. Aschwanden $(\triangle)$ \\ e-mail: aschwanden@1msal.com \\ L. F. Burlaga · M. L. Kaiser · C. K. Ng · D. V. Reames · M. J. Reiner \\ NASA/Goddard Space Flight Center, Greenbelt, MD 20771, USA
}

Solar \& Astrophysics Lab., Lockheed Martin ATC, 3251 Hanover St., Palo Alto, CA 94304, USA

T. I. Gombosi · N. Lugaz · W. Manchester IV · I. I. Roussev · T. H. Zurbuchen University of Michigan, 1141 Space Research Bldg., Ann Arbor, MI 48109, USA

C. J. Farrugia - A. B. Galvin · M. A. Lee

University of New Hampshire, EOS SSC Morse Hall, Durham, NH 03824, USA

J. A. Linker · Z. Mikić · P. Riley

SAIC, 10260 Campus Point Dr., San Diego, CA 92121, USA

D. Alexander · A. W. Sandman

Dept. Physics \& Astronomy, Rice University, PO Box 1892, Houston, TX 77251, USA

J. W. Cook · R. A. Howard

Naval Research Laboratory, Code 7660, 4555 Overlook Ave. SW, Washington DC, 20375, USA

D. Odstrčil · V. J. Pizzo

NOAA/SEC, Code R/E/SE, 325 Broadway, Boulder, CO 80303, USA

J. Kóta

University of Arizona, Lunar and Planetary Laboratory, Tucson, AZ 85721-0092, USA 
instruments (IMPACT, PLASTIC). The modeling includes the coronal plasma, in both open and closed magnetic structures, and the solar wind and its expansion outwards from the Sun, which defines the heliosphere. Particular emphasis is given to modeling of dynamic phenomena associated with the initiation and propagation of coronal mass ejections (CMEs). The modeling of the CME initiation includes magnetic shearing, kink instability, filament eruption, and magnetic reconnection in the flaring lower corona. The modeling of CME propagation entails interplanetary shocks, interplanetary particle beams, solar energetic particles (SEPs), geoeffective connections, and space weather. This review describes mostly existing models of groups that have committed their work to the STEREO mission, but is by no means exhaustive or comprehensive regarding alternative theoretical approaches.

Keywords STEREO mission · Solar corona - Solar wind - Coronal mass ejection (CME) · Solar filaments $\cdot$ Heliosphere $\cdot$ Interplanetary shocks $\cdot$ Interplanetary particle beams $\cdot$ Solar energetic particle events (SEP) · Solar flares $\cdot$ Space weather $\cdot$ Stereoscopy $\cdot 3 D$ reconstruction techniques $\cdot$ White-light emission $\cdot$ EUV emission · Interplanetary radio emission

P. C. Liewer

Jet Propulsion Laboratory, Mail Stop 169-506, Pasadena, CA 91109, USA

J. G. Luhmann

University of Berkeley, Space Sciences Lab., 7 Gauss Way \#7450, Berkeley, CA 94720, USA

B. Inhester · R. W. Schwenn · S. K. Solanki · V. M. Vasyliunas · T. Wiegelmann

Max-Planck Institut für Sonnensystemforschung, Max-Planck Str.2, D-37191, Katlenburg-Lindau,

Germany

L. Blush · P. Bochsler

Physikalisches Institut,University of Bern, Sidlerstrasse 5, CH-3012 Bern, Switzerland

I. H. Cairns · P. A. Robinson

University of Sydney, School of Physics, Sydney, NSW 2006, Australia

V. Bothmer

Universität Göttingen, Institut für Astrophysik, Friedrich-Hund-Platz 1, 37077 Göttingen, Germany

K. Kecskemety

KFKI RMKI, POB 49, H-1525, Budapest, Hungary

A. Llebaria

Laboratoire d'Astronomie Spatiale, C.N.R.S., BP8, 13376 12, Marseille Cedex, France

M. Maksimovic

CNRS \& LESIA, Observatore de Paris-Meudon, F-92195 Meudon Cedex, France

M. Scholer

Center for Interdisciplinary Plasma Science, Max-Planck-Institute for Extraterrestrial Physics, 85740 Garching, Munich, Germany

R. F. Wimmer-Schweingruber

Extraterr, Physics IEAP, University of Kiel, Leibnizstr. 11, Kiel, 24118 Germany

은 Springer 


\section{Introduction}

Theoretical modeling is of particular importance for the Solar TErrestrial RElations $\mathrm{Ob}$ servatory (STEREO) mission because we obtain for the first time mission-dedicated 3dimensional (3D) information of solar-terrestrial phenomena. Some 3D modeling has been accomplished before, of course, e.g., by means of solar-rotation tomography, combined imaging and Doppler-spectroscopy, or multiple in-situ spacecraft observations of the solar wind (such as with CLUSTER), but STEREO is the first mission dedicated to study the 3D evolution of CMEs and the solar wind from stereoscopic vantage points. Realistic models of the 3D structure and dynamics of solar/heliospheric plasma and particles are needed to fully exploit the science return of the data, for our exploration of the unknown physical processes whose outcome we are observing, as envisioned in a number of anticipatory pre-launch papers (Grigoryev, 1993; Pizzo et al., 1994; Davila et al., 1996; Schmidt and Bothmer, 1996; Socker et al., 1996, 2000; Rust et al., 1997; Socker, 1998; Liewer et al., 1998; Howard et al., 2002; Davila and St. Cyr 2002; Mueller et al., 2003). Table 1 gives an overview of what the four instrument suites of the two STEREO spacecraft will yield: SECCHI/EUVI will image the solar corona, eruptive filaments, flares, and coronal mass ejections (CMEs) in the lower corona at EUV wavelengths. SECCHI/COR and HI will image the CME phenomena that propagate to the outer corona in white light. SWAVES will triangulate the radio emission generated by CMEs and interplanetary shocks and particle beams. The IMPACT and PLASTIC instruments are in-situ particle detectors that measure particle distribution functions and elemental abundances at $1 \mathrm{AU}$ in the solar wind or in passing CMEs, interplanetary shocks, particle beams, or in solar energetic particle (SEP) events. The theoretical modeling of all these processes includes both magneto-hydrodynamic (MHD) and kinetic theories. A great potential, but also challenge, is the unprecedented computer power that supports these theoretical and numerical modeling efforts today, never available to such a large extent in previous missions.

We organize this review in the following order: First we describe theoretical modeling of the solar/heliospheric background plasma (solar corona in Section 2, solar

Table 1 Metrics of modeled solar/heliospheric phenomena versus detecting STEREO instruments

\begin{tabular}{|c|c|c|c|c|}
\hline & $\begin{array}{l}\text { SECCHI } \\
\text { EUVI, COR /HI }\end{array}$ & SWAVES & IMPACT $^{\mathrm{a}}$ & PLASTIC \\
\hline \multicolumn{5}{|l|}{ Background plasma } \\
\hline Solar corona (Section 2) & EUV, WL & $\ldots$ & $\ldots$ & $\ldots$ \\
\hline Solar wind (Section 3) & $\ldots$ & Waves & Particles & Particles \\
\hline \multicolumn{5}{|l|}{ CME Initiation } \\
\hline Filament eruption (Section 4) & EUV, WL & $\ldots$ & $\ldots$ & $\ldots$ \\
\hline $\begin{array}{l}\text { Coronal mass ejection launch } \\
\text { (Section 5) }\end{array}$ & EUV, WL & Radio, waves & $\ldots$ & $\ldots$ \\
\hline \multicolumn{5}{|l|}{ CME Propagation } \\
\hline Interplanetary shocks (Section 6) & WL & Radio, waves & Particles & Particles \\
\hline $\begin{array}{l}\text { Interplanetary particle beams } \\
\quad(\text { Section } 7)\end{array}$ & $\ldots$ & Radio, waves & Particles & Particles \\
\hline $\begin{array}{l}\text { Solar energetic particle events } \\
\quad(\text { Section } 8)\end{array}$ & $\cdots$ & $\ldots$ & Particles & Particles \\
\hline $\begin{array}{l}\text { Geo-connected space weather } \\
\quad \text { (Section 9) }\end{array}$ & $\cdots$ & $\ldots$ & Particles & Particles \\
\hline
\end{tabular}

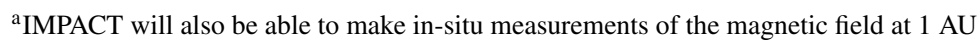


wind in Section 3), then processes of CME initiation (filament eruption in Section 4, CME launch in Section 5), and finally processes of interplanetary CME propagation (interplanetary shocks in Section 6, interplanetary particle beams and radio emission in Section 7, solar energetic particles in Section 8, geoeffective events and space weather in Section 9).

\section{Modeling of the Solar Corona}

\subsection{Physical 3D-Modeling of the Global Corona}

The quantitative analysis of stereoscopic EUV images requires full 3D models of the electron density $n_{e}(x, y, z)$ and electron temperature $T_{e}(x, y, z)$ of the coronal plasma, so that emission measure images $E M(x, y)$ can be self-consistently produced by integrating the differential emission measure (DEM) distribution, i.e., $d E M(x, y, T) / d T=\int n_{e}^{2}(x, y, z, T) d z$, along each stereoscopic line-of-sight direction $z$. In addition, the DEM depends also on assumptions on elemental abundances and ionization equilibrium (see Section 5.2 for more details and references). The most detailed state-of-the-art models represent the inhomogeneous 3D solar corona with up to $\approx 10^{5}$ coronal loop structures, each one calculated based on a physical model (e.g., Schrijver et al., 2004). A key observable input is a (synoptic) full-Sun magnetogram of the photospheric magnetic field as a boundary condition, which can be extrapolated into the 3D corona by means of a potential field (source surface) model or a nonlinear force-free field model. An energy input into the corona has to be assumed, which could be a function of the local magnetic field strength $B(x, y)$ at the footpoint and the loop length $L(x, y)$, yielding a local Poynting flux (or heating rate) of $E_{H}(x, y) \propto B^{a}(x, y) L^{b}(x, y)$ at position $(x, y)$. The physical model of a coronal loop can then be specified by a hydrostatic equilibrium solution, where the heating rate is balanced by the conductive and radiative losses, e.g., the RTV solutions known for uniform heating and constant pressure (Rosner et al., 1978), the RTVS solutions corrected for non-uniform heating and gravitation (Serio et al., 1981), or empirical scaling laws inferred from Yohkoh observations (Kano and Tsuneta, 1995). The latest TRACE studies imply deviations from the equilibrium scaling laws because of the asymmetric heating functions caused by flows (Winebarger et al., 2002). The emission measures $d E M(x, y, z, T) / d T$ of the physical loops can then be filled into a datacube $(x, y, z)$ aligned with a (stereoscopic) direction $z$ and integrated along this line-of-sight. Full-Sun visualizations based on such physical models have been simulated for soft X-ray and EUV instruments (Figure 1). The input parameters (such as the magnetic field model or the heating scaling law) can then be varied until the simulated images show the best match (quantified by a $\chi^{2}$-value) with an observed soft X-ray or EUV image. Fitting two stereoscopic EUV images from SECCHI/EUVI simultaneously with the same physical 3D model obviously represents a very powerful method to constrain the heating function, a key observable in the coronal heating problem.

The $3 \mathrm{D}$ reconstructions of the magnetic field and electron density of the global corona have been attempted for decades (e.g., Altschuler, 1979): from line-of-sight inversions of the white-light polarization (e.g., Van de Hulst, 1950; Lamy et al., 1997; Llebaria et al., 1999; Quémerais and Lamy, 2002), from synoptic maps combined with magnetic field extrapolations (Liewer et al., 2001), from stereoscopic image pairs in soft X-rays (Batchelor, 1994), from stereoscopic or multi-frequency images in radio (Aschwanden and Bastian, 1994a,b; Aschwanden et al., 1995, 2004; Aschwanden, 1995), from tomographic multi-image sequences in soft X-rays or EUV (Hurlburt et al., 1994; Davila, 1994; Zidowitz et al., 1996; 


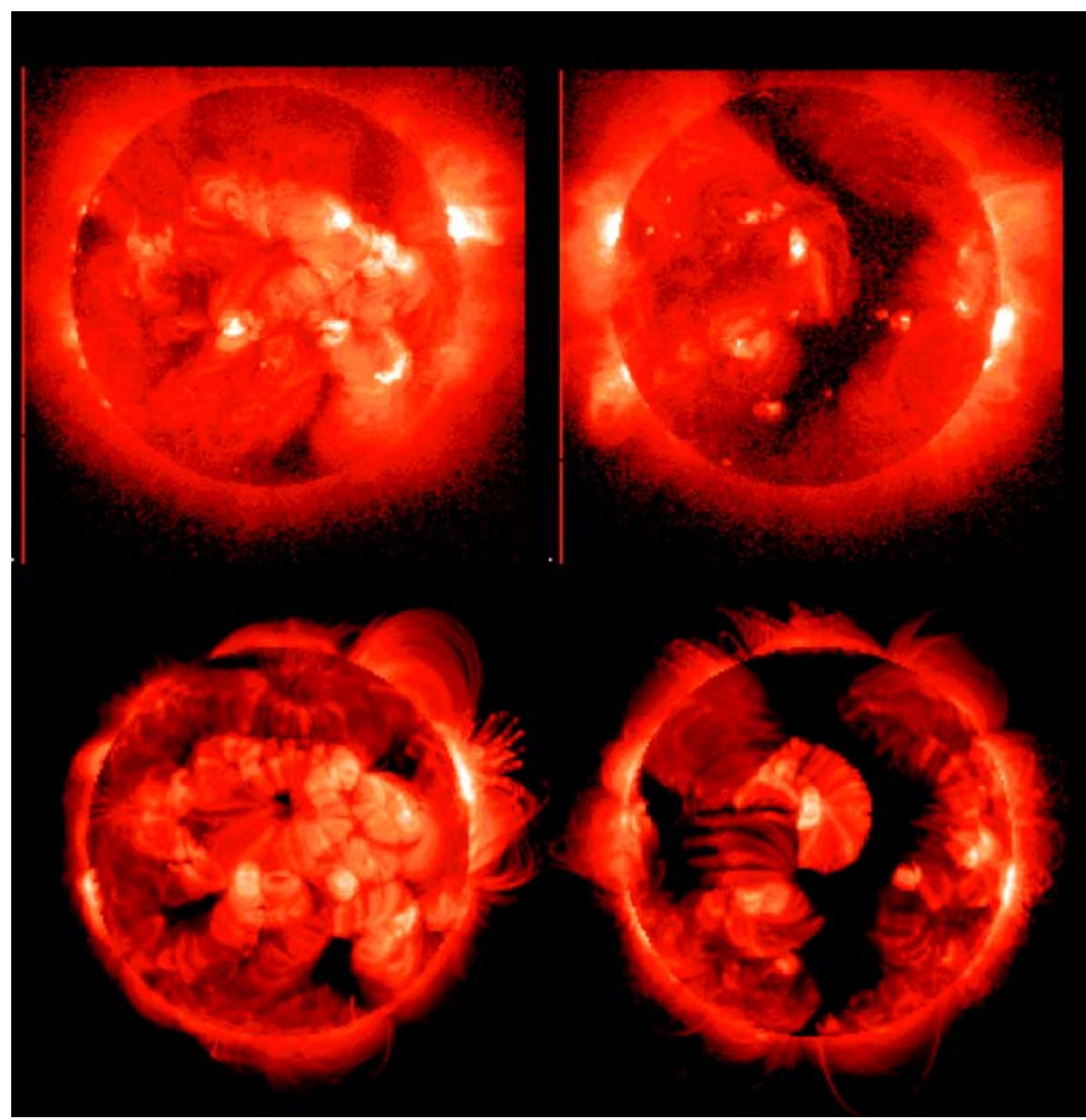

Fig. 1 Full-Sun visualization of stereoscopic corona modeling: soft X-ray images from Yohkoh/SXT from two different aspect angles (top row), and simulated 3D corona images (bottom row), both shown on a logarithmic scale with a total range of 4 orders of magnitude in brightness. The theoretical 3D model is based on the observed magnetic field on the solar surface, a potential magnetic field model, a heating function, hydrostatic solutions of $\approx 50,000$ individual coronal loops, and convolution with the filter response functions (Schrijver et al., 2004)

Zidowitz, 1997, 1999; Frazin and Kamalabadi, 2005), or from DEM-tomographic multi-filter images (Frazin, 2000; Frazin and Janzen, 2002; Frazin et al., 2005). However, these reconstructions of the 3D density $n_{e}(x, y, z)$ of the global corona have only been demonstrated with a resolution of $\gtrsim 15^{\circ}$ in longitude, mostly limited by the time variability over the timespan of substantial rotation. Such approaches can characterize the smooth 3D density of the background corona, but cannot be used to reconstruct elementary coronal loop structures (which require a spatial resolution of $\lesssim 1^{\prime \prime}$ ). However, some numerical simulation studies have zoomed into partial views of the $3 \mathrm{D}$ corona, rendering active regions on the level of elementary loops, based on hydrodynamic loop models (Gary, 1997; Alexander et al., 1998) or full-scale MHD simulations with realistic plasma heating from photospheric drivers (Gudiksen and Nordlund, 2002, 2005a,b; Mok et al., 2005). 


\subsection{Stereoscopic 3D-Reconstruction of Coronal Loops}

Although stereoscopic observations with two spacecraft provide only limited constraints for $3 \mathrm{D}$ modeling of the global corona, the $3 \mathrm{D}$ reconstruction of a single elementary loop structure should be much better constrained, if we succeed to isolate a single loop by appropriate subtraction of the background corona, which consists of myriads of other competing loop structures. 3D reconstructions of elementary loop structures are of fundamental importance for studying the physical plasma properties, their (MHD) dynamics, the associated (non-potential) magnetic field and electric currents (e.g., Aschwanden, 2004, Sections 3-8). The mathematical determination of the 3D geometry of a single loop has been formulated for planar loops (Loughhead et al., 1983) as well as for non-planar loops (Berton and Sakurai, 1985). The determination of the 3D position of a point-like feature, such as the loop centroid in a particular viewing plane, is essentially a triangulation method in epipolar planes (Portier-Fozzani and Inhester, 2001, 2002), also called tiepoint method (Figure 2) in some applications to solar stereoscopy (Liewer et al., 2000; Hall et al., 2004).

Such stereoscopic 3D reconstructions of single loops have been attempted in the past by using the solar rotation to mimic two different viewing angles, which of course works only for stationary loops. 3D reconstructions of single coronal structures (threads, rays,
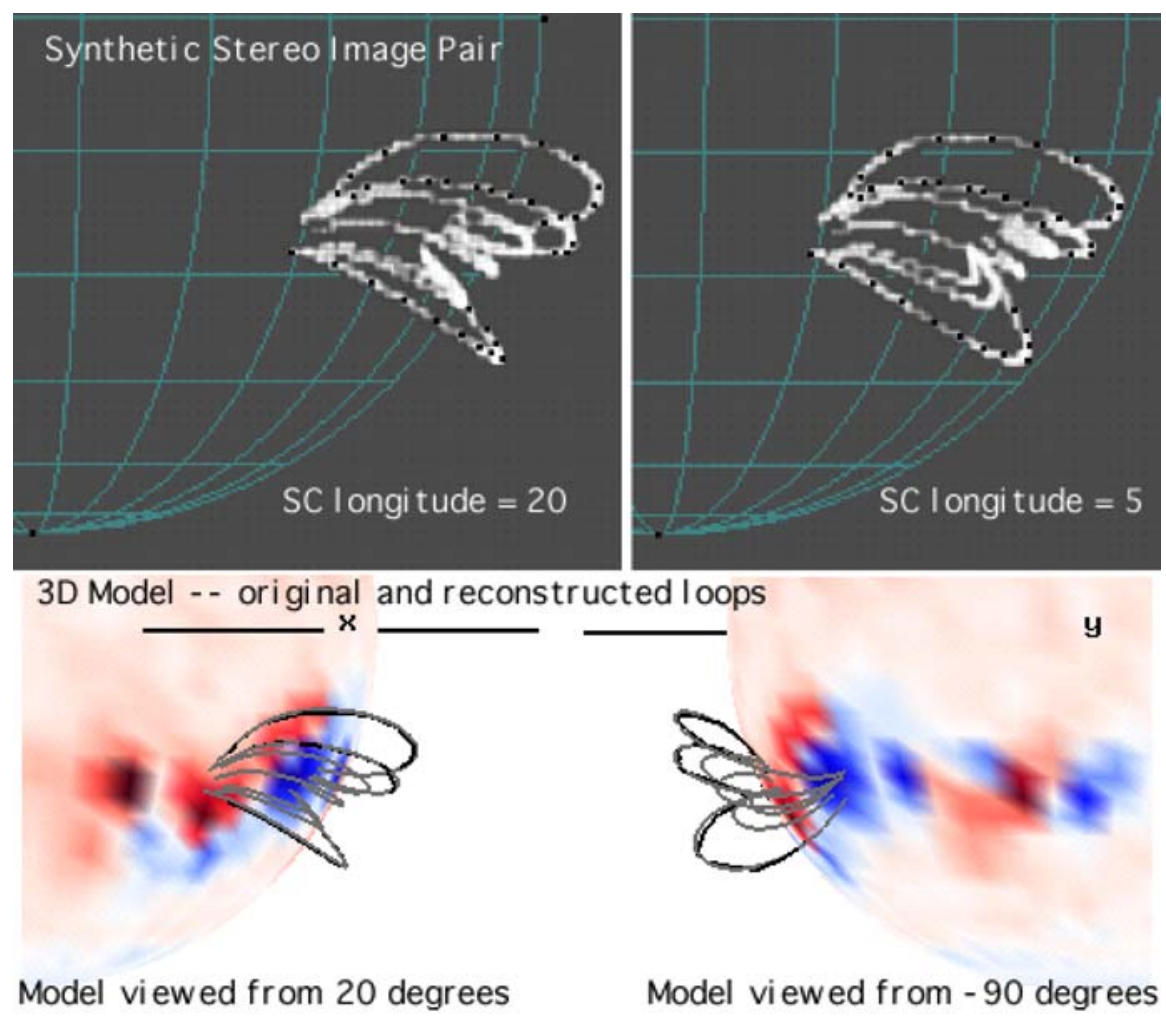

Fig. 2 Stereoscopic 3D reconstruction of individual loops in EUV images using the tie-point method. Two corresponding loop structures have to be identified in a pair of images, pinpointed with tiepoints for triangulation of their 3D geometry (courtesy of Eric DeJong and Paulett Liewer) 
streamers) aligned with individual coronal magnetic field lines have been achieved from white-light images taken 1-3 h apart (Koutchmy and Molodensky, 1992; Vedenov et al., 2000). In order to make solar-rotation stereoscopy more general, the concept of dynamic stereoscopy has been developed for the 3D reconstruction of coronal loops, which relies more on static magnetic fields, rather than on static brightness maps (Aschwanden et al., 1999, 2000). Alternative 3D reconstructions of magnetic field lines combine theoretical $3 \mathrm{D}$ magnetic field models with the observed 2D projection of a coronal loop from an EUV image (Gary and Alexander, 1999; Wiegelmann and Neukirch, 2002; Wiegelmann and Inhester, 2003; Wiegelmann et al., 2005), which can be even more strongly constrained by two simultaneous projections from two STEREO spacecraft (Wiegelmann and Inhester, 2006). Forward-fitting techniques using some a priori constraints are expected to be superior to straightforward backprojection techniques (Gary et al., 1998). The efficiency of stereoscopic correlations can be considerably enhanced with automated detection of loops, e.g., with the oriented-connectivity method (Lee et al., 2005; Aschwanden, 2005), with help of extrapolated magnetic field lines (Wiegelmann et al., 2005), or even by constraining the heating input with subsurface (magnetoconvection) dynamics (Hurlburt et al., 2002). Stereoscopy of coronal loops is expected to be most suitable at small separation angles $\left(\lesssim 30^{\circ}\right)$, which has to take place in the initial phase (during the first year) of the STEREO mission.

\section{Modeling of the Solar Wind}

In order to understand the propagation of CMEs and energetic particles from the corona through the heliosphere, detailed time-dependent models of the background plasma and solar wind are required. Solar wind models can be subdivided depending on their boundary conditions, either given by the magnetic field in the lower corona (Section 3.1), or by heliospheric conditions (Section 3.2). Recent space weather models involve the fully connected Sun-to-Earth system by coupling in with magnetospheric and ionospheric models, such as in the Community Coordinated Modeling Center (CCMC), and these will provide the most comprehensive context for STEREO data.

\subsection{Coronal Solar Wind Models}

An approximate description of the global coronal magnetic field close to the Sun is given by the so-called potential field source surface (PFSS) model, constrained by the lower boundary condition of the photospheric magnetic field and an upper artificial boundary condition at $r \approx 1.6-3.25 R_{\odot}$, where the magnetic field is assumed to be current-free $(\nabla \times \mathbf{B}=0)$. There exist a number or numerical codes based on such PFSS models, initially developed by Altschuler and Newkirk (1969) and Schatten et al. (1969), later refined by Hoeksema (1984) and Wang and Sheeley (1992), and recently used with input from Wilcox Solar Observatory magnetograms (at CCMC), or from Michelson Doppler Imager (MDI) magnetograms onboard the Solar and Heliospheric Observatory (SoHO) (Schrijver and DeRosa, 2003). These codes are extremely useful to map out open magnetic field regions that connect not only from coronal holes but also from some parts of active regions out into the heliosphere (Figure 3), outlining escape paths for high-energetic particles.

The Magnetohydrodynamics Around a Sphere (MAS) model is developed by the Science Applications International Corporation (SAIC) group, which is an MHD model of the solar corona extending over a domain of 1-30 solar radii. The input of the model is (1) the radial 

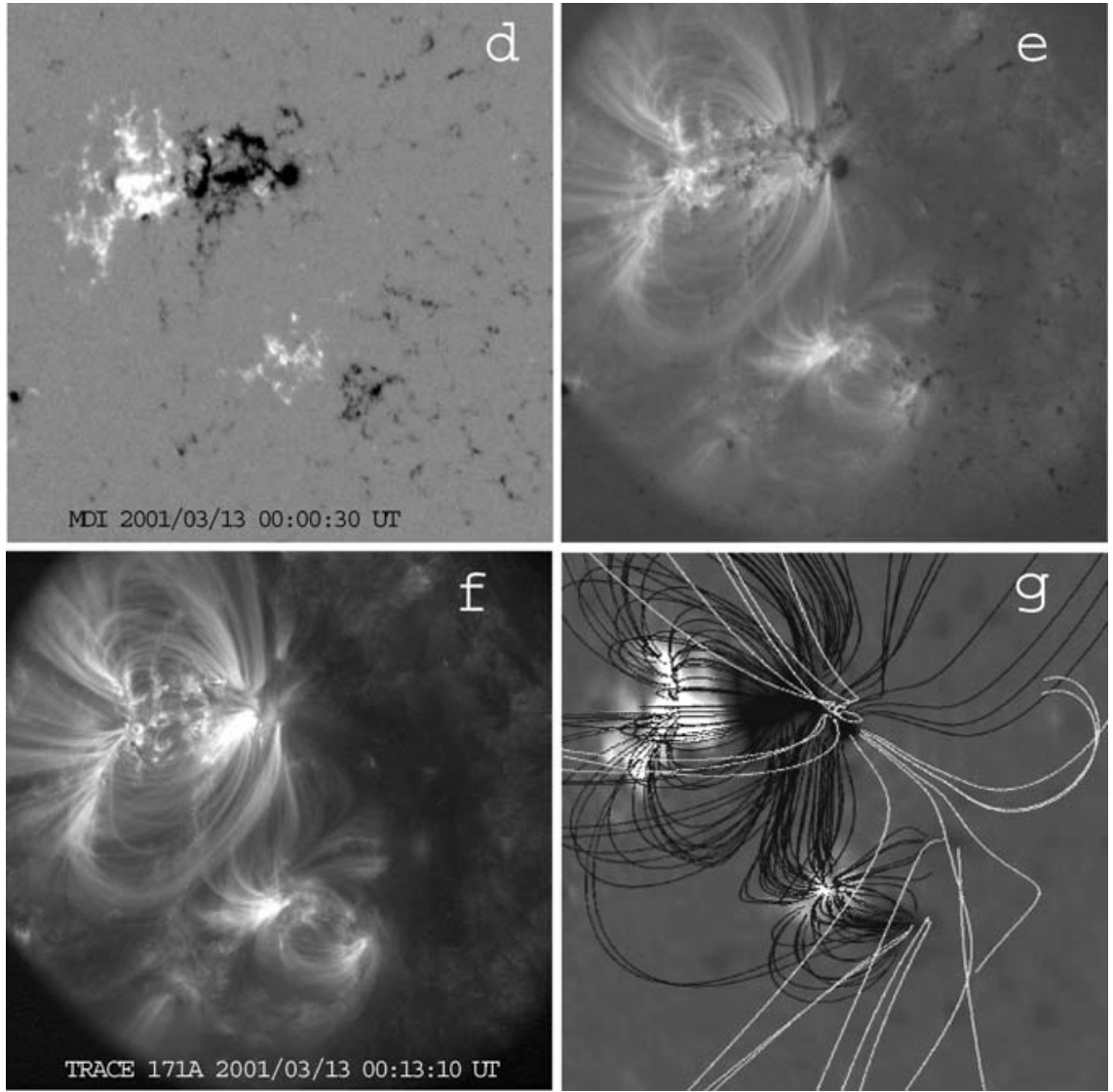

Fig. 3 (Panel d) MDI magnetogram; (Panel e) MDI magnetogram overlayed with TRACE $171 \AA$; (Panel f) TRACE $171 \AA$ A image of 2001-Mar-13,00:13 UT; (Panel $g$ ) Potential field extrapolation using a source-surface model. Closed field lines of active regions are indicated with black color, the open field lines that connect to interplanetary space with white color (Schrijver and DeRosa, 2003)

magnetic field $B_{r}(\vartheta, \phi)$ as a function of co-latitude $\vartheta$ and longitude $\phi$ from a (full-Sun) synoptic magnetogram (e.g., from Kitt Peak National Observatory, KPNO) that is slightly smoothed, and (2) the temperature $T_{e}(\vartheta, \phi)$ and density $n_{e}(\vartheta, \phi)$ at the coronal base. The model computes a stationary solution of the resistive MHD equations and provides as output the plasma temperature $T_{e}(r, \vartheta, \phi)$, pressure $p(r, \vartheta, \phi)$, density $n_{e}(r, \vartheta, \phi)$, solar wind velocity $v(r, \vartheta, \phi)$, and magnetic field $B(r, \vartheta, \phi)$ as a function of the distance, in the range of $1<$ $r<30 R_{\odot}$. The initial condition employs a transonic solution for the gas-dynamic variables with a polytropic index of $\gamma=1.05$, which avoids the complications of the heating, thermal conduction, and radiative loss terms in the energy equation. An example of such a 3D model is shown in Figure 4. The MAS model has been used to simulate 3D coronal streamers (Linker et al., 1990) and the solar corona during the whole-Sun month (Linker et al., 1999). Given an (approximate) 3D model of the coronal density, stereoscopic images in white-light can be integrated straightforwardly and compared with observed images from SECCHI/COR and HI. 


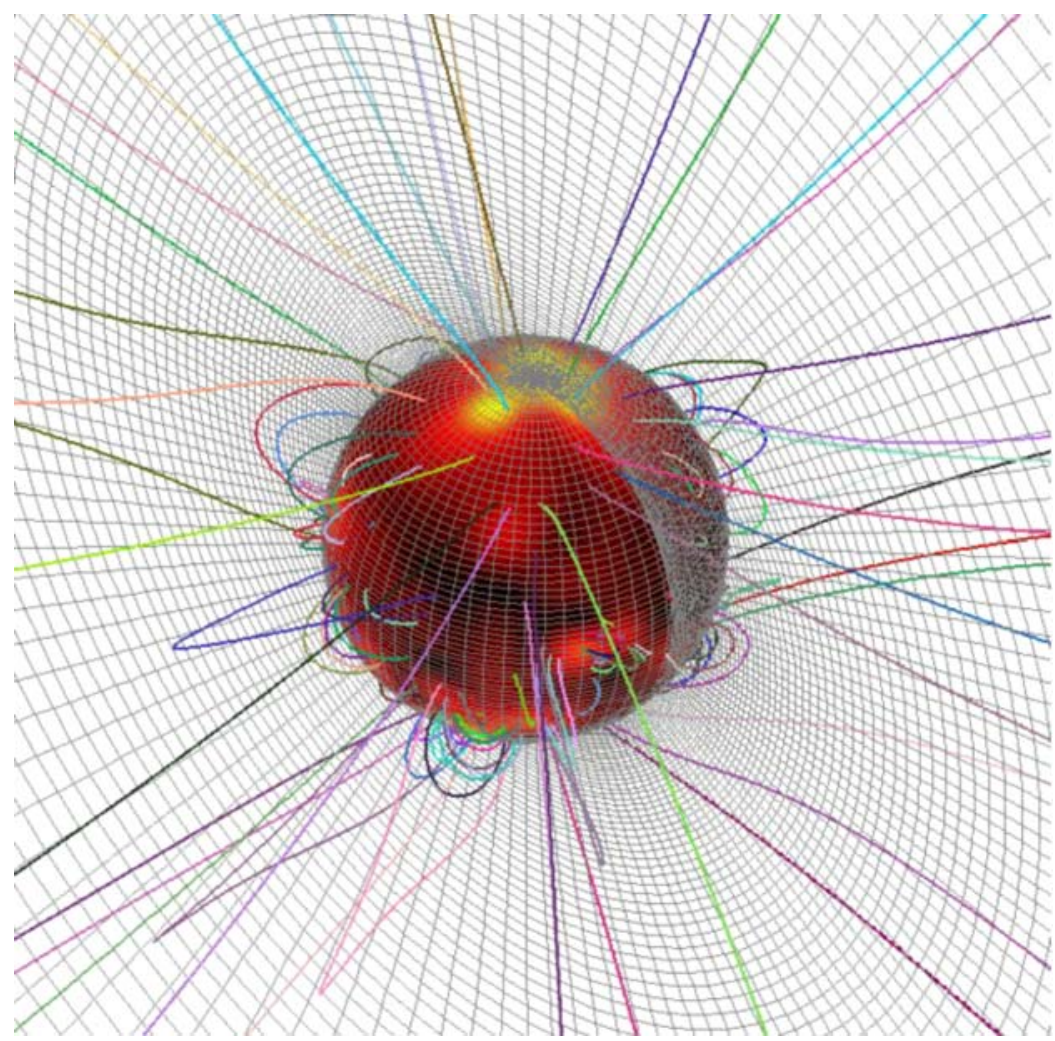

Fig. 4 A 3D numerical computation of the solar corona with $101 \times 75 \times 64$ (non-uniform) meshpoints $(r, \vartheta, \phi)$, (courtesy of SAIC group)

\subsection{Heliospheric Solar Wind Models}

Recent numerical codes that simulate or reconstruct the solar wind in the heliosphere (e.g., Schwenn and Marsch, 1991a,b; Neugebauer, 2001; Balogh et al., 2001) include MAS-IP (Riley et al., 2001a,b), ENLIL (developed by D. Odstrčil), heliospheric tomography (developed by B. Jackson and P. Hick), and the exospheric solar wind model (developed by H. Lamy and V. Pierrard), all part of the space weather modeling effort coordinated by CCMC. The aim of these codes is to provide components for "end-to-end models" (e.g., CISM, UMich, and CCMC) that link the coronal and solar wind physics and geometry - which is just what the STEREO combined imaging and in-situ experiments are trying to do.

In the past we have had many separate coronal/imaging studies and on the other side in-situ studies. But linking them demands combined data sets and coupled corona/solar-wind models with realistic characteristics. For example, the magnetic field models tell us for a particular photospheric boundary condition where open field regions (hence solar wind sources) should be located, and which ones connect to specific points in space, e.g., to the STEREO spacecraft or to the Earth (within the uncertainty of the chosen magnetic field model, of course). So we may associate a particular coronal hole seen in an EUV image with a solar wind stream we detect on the spacecraft or at Earth. 


\section{The Heliosphere During Whole Sun Month}

\section{August - September 1996}

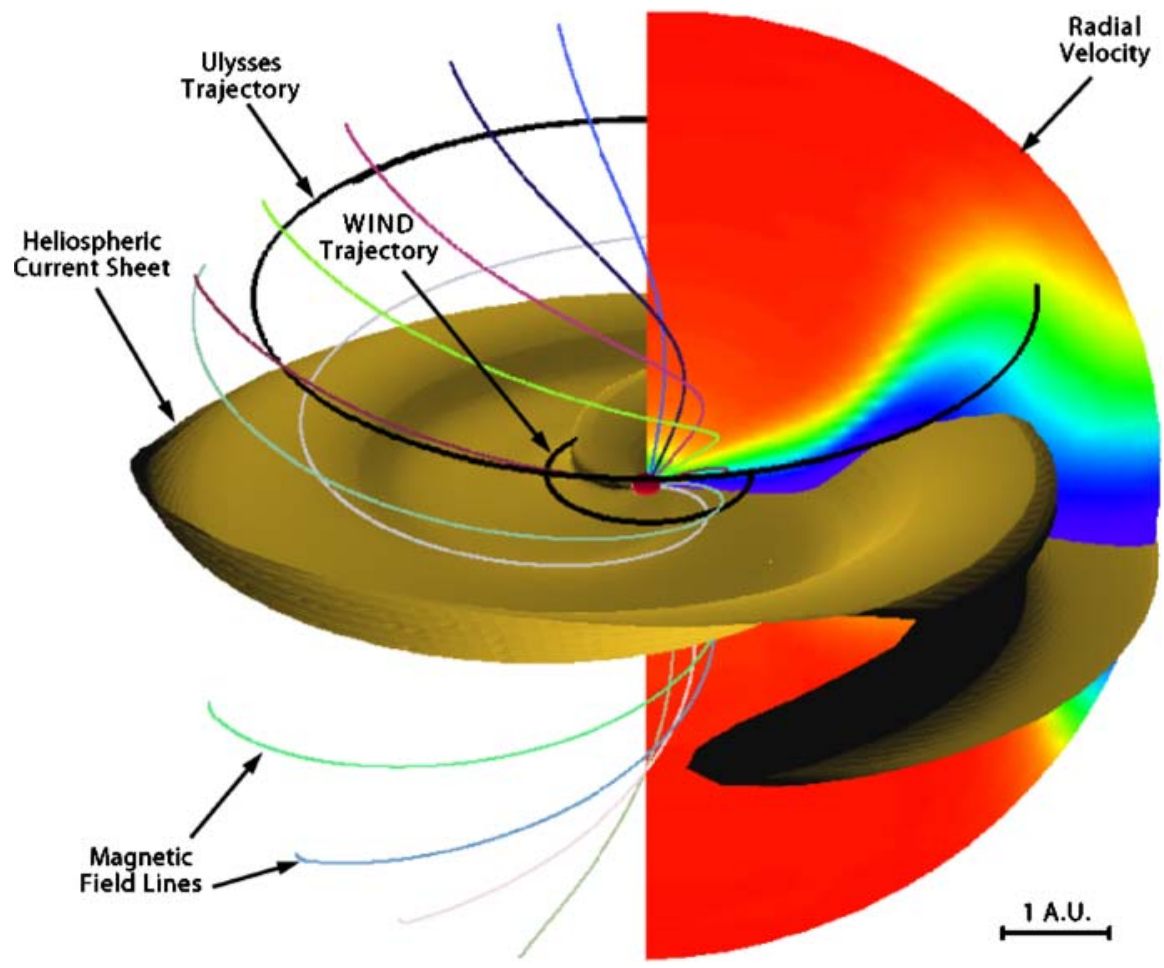

Fig. 5 Model solution for Carrington rotations (CR) 1912-1913. The heliospheric current sheet (inferred from the isosurface $B_{r}=0$ ) is displayed out to $5 \mathrm{AU}$. The central sphere marks the inner boundary at $30 R_{\odot}$. A meridional slice of the radial velocity is shown at an arbitrary longitude. Red corresponds to fastest speeds $\left(\approx 750 \mathrm{~km} \mathrm{~s}^{-1}\right)$ and blue to the slowest speeds $\left(\approx 350 \mathrm{~km} \mathrm{~s}^{-1}\right)$. Superimposed is a selection of interplanetary magnetic field lines originating from different latitudes. Finally, the trajectories of the Wind and Ulysses spacecraft are marked (Riley et al., 2001b)

The solar physics group at SAIC has developed a 3D MHD model of the solar corona and heliosphere (Riley et al., 2001a,b). They split the modeling region into two distinct parts: the solar corona $\left(1-30 R_{\odot}\right)$ and the inner heliosphere $\left(30 R_{\odot}-5 \mathrm{AU}\right)$. The combined model is driven solely by the observed line-of-sight photospheric magnetic field and can thus provide a realistic global picture of the corona and heliosphere for specific time periods of interest. Figure 5 summarizes the global structure of the inner heliosphere for the interval coinciding with Carrington rotation CR 1913 (1996 August 22-1996 September 18), which occurred near solar minimum and overlapped the first "Whole Sun Month" campaign. Comparisons of Ulysses and Wind observations with the simulation results for a variety of time periods (e.g., Riley et al., 2003a) show that the model can reproduce the overall features of observations. In a subsequent study, the SAIC team employed this model to explore the evolution of the heliospheric current sheet (HCS) during the course of the solar cycle (Riley et al., 2002a). They compared their results with a simple "constant-speed" approach for mapping the HCS outward into the solar wind, demonstrating that dynamic effects can substantially deform the HCS in the inner heliosphere $(\lesssim 5 \mathrm{AU})$. They also noted that while the HCS may almost 
always be topologically equivalent to a "ballerina skirt", more complicated shapes were possible. One example was an interval approaching the maximum of solar cycle 23 (CR 1960 and 1961) when the shape would be better described as "conch shell"-like.

The NOAA/SEC heliospheric model (ENLIL) solves the time-dependent MHD equations in a spherical geometry using either the Flux-Corrected-Transport or Total-VariationDiminishing schemes (e.g., Odstrčil, 1994; Toth and Odstrčil, 1996, 2003; Odstrčil et al., 2002). These high-resolution schemes produce second-order accuracy away from discontinuities, while simultaneously providing the stability that ensures non-oscillatory solutions. The inner radial boundary is located beyond the sonic point $\left(\approx 21.5-30 R_{\odot}\right)$, provided, e.g., by the MAS or Wang-Sheeley-Arge (WSA) code. The outer radial boundary can be adjusted to $1-10 \mathrm{AU}$, and the latitudinal extent covers $\approx \pm 60^{\circ}$ north and south of the ecliptic.

In support of the STEREO mission, the CCMC is running a series of solar and heliospheric models (by coupling the MAS and ENLIL code) and is saving model input/output on a daily basis. Driven by synoptic magnetogram data obtained by ground-based solar observatories, the solar coronal potential field source surface (PFSS) model represents the approximate coronal magnetic field within $2.5 R_{\odot}$. The ENLIL solar wind is driven by the WSA model (Arge and Pizzo, 2000) which extends a PFSS magnetic field to $21.5 R_{\odot}$ past the sonic point (where the plasma velocity starts to exceed the sound speed) using a heliospheric current sheet model and a slow and high speed solar wind distribution depending on the location of coronal holes. ENLIL covers the radial distance between the WSA boundary of $21.5 R_{\odot}$ and 1.6 AU in the inner heliosphere, between $\pm 58^{\circ}$ degrees heliographic latitude (which brackets the streamer regions).

In both the PFSS and ENLIL models the time stamp of each file refers to the end time of the solar rotation period covered by the magnetogram data. Typically this date lies about 2 days in the future, as magnetic fields on the solar disc can be measured fairly reliably up to 30 degrees of heliographic longitude away from the disk center (Carrington longitude of the Earth).

The heliospheric tomography model makes use of interplanetary scintillation (IPS) data to tomographically reconstruct the global structure of the solar wind, provided by earlier IPS observations from STELab in Nagoya, Japan. The model ouptut yields solar wind density and velocity throughout the inner heliosphere, and is able to make real-time heliospheric 3D reconstructions (Jackson and Hick, 2002). For a review of solar wind properties from IPS observations, see, e.g., Kojima et al. (2004). Since January 2003, the Solar Mass Ejection Imager Mission (SMEI) has been providing data for the IPS Thomson scattering modeling of the all-sky heliospheric solar wind and CMEs (Figure 6).

The 1-D exospheric solar wind model (Lamy et al., 2003), also part of the CCMC end-toend model chain, is developed for coronal holes over a radial range of $\approx 2-30 R_{\odot}$, including protons and electrons, modeled with a nonmonotonic total potential for the protons, and with a Lorentzian (kappa) velocity distribution function for the electrons. The exospheric kinetic model assumes that there is a critical height where there is a transition from a collisiondominated to a collisionless regime (at $\approx 1.1-5.0 R_{\odot}$, called the exobase). An overview of the main differences between the exospheric and fluid/MHD approaches is given in Cranmer (2002).

In addition to the CCMC effort, numeric codes to simulate the steady-state solar wind with helmet-type streamer belt have been developed by the MHD modeling group at the University of Michigan. An example of such a 3D MHD simulation is shown in Roussev et al. (2003a), designed to reproduce the global structure of the solar corona and wind under realistic conditions. The magnetic field in the model is split into a potential, $\mathbf{B}_{0}$, 


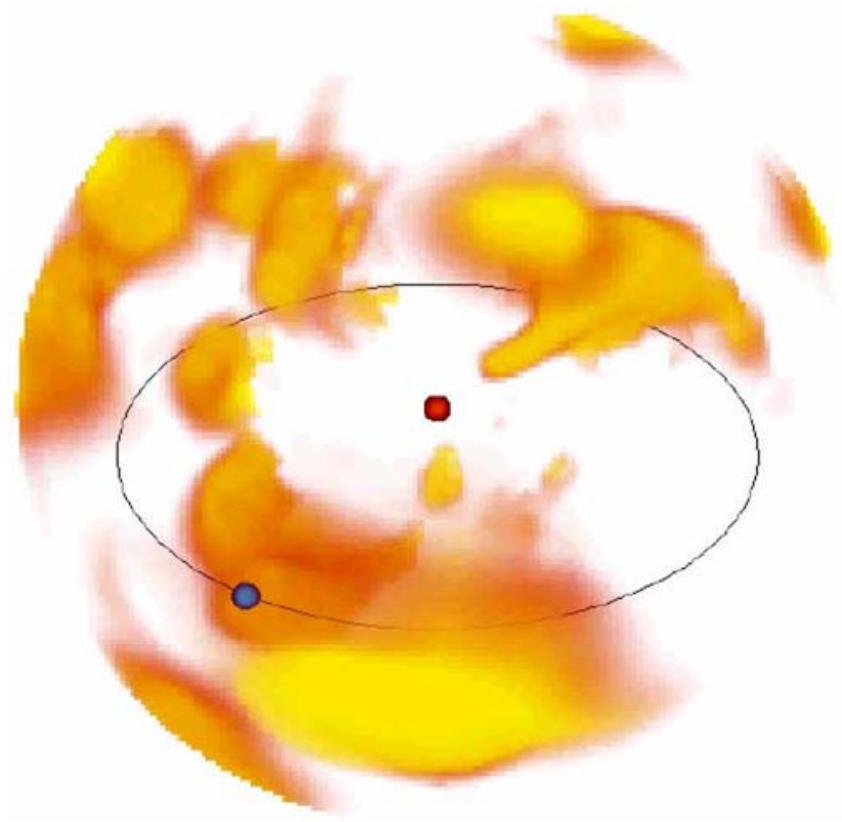

Fig. 6 Time-dependent tomographic reconstruction of the solar wind using SMEI data during the 2003 May $28 \mathrm{CME}$ (courtesy of Bernie Jackson)

and a non-potential, $\mathbf{B}_{1}$, part: $\mathbf{B}=\mathbf{B}_{0}+\mathbf{B}_{1}$, where $\nabla \times \mathbf{B}_{0}=0$. To obtain the bulk solar magnetic field, $\mathbf{B}_{0}=-\nabla \psi$, the PFSS method by Altschuler et al. (1977) is used. In this method, the magnetic scalar potential, $\psi$, is evaluated as a series of spherical harmonics. The coefficients in the series are chosen to fit real magnetogram data obtained from the Wilcox Solar Observatory, and most recently from SoHO/MDI. The MHD solution in the model is evolved from a static, potential initial configuration to a steady-state, non-potential solution with a non-zero induced field, $\mathbf{B}_{1}$. The solar wind is powered (heated and accelerated) by the energy interchange between the solar plasma and large-scale MHD turbulence, assuming that the additional energy is stored in the "turbulent" internal degrees of freedom. Note that close to the Sun, an additional amount of energy is stored in waves and turbulent fluctuations, hence the specific heat ratio, $\gamma$, of the solar plasma is close to 1 (e.g., Steinolfson and Hundhausen, 1988). The lower values of $\gamma$ near the Sun are assumed to be associated with those "turbulent" internal degrees of freedom. It is assumed that $n=n_{0}+n_{\text {turb }}(R)$, where the number of "turbulent" degrees of freedom near the Sun is $n_{\text {turb }}\left(R_{\odot}\right) \approx 10$, while at larger distances it drops to zero, i.e., $n_{\text {turb }}(\infty) \approx 0$, similar to the approach described in Zeldovich and Raizer (2002) for partially ionized plasmas. Specifically, $n_{\text {turb }}(R)=10(R \odot / R)^{m}$, with $m=1$ is assumed in the original work by Roussev et al. (2003a). Thus the full energy equation is employed in the computations, with a polytropic index $\gamma(R)=[n(R)+2] / n(R)$ that is now a function of radius describing the additional energy density associated with turbulent motions. This technique is an empirical one inspired by the "hidden internal" degrees of freedom. The physical motivation is to bridge a polytrope, which is nearly isothermal, to a fully fledged energy equation.

The 3D models of the corona and solar wind described above will help to link IMPACT solar wind measurements to the Sun by allowing observations of specific electron populations, 
magnetic fields, and solar flare particle events to be mapped back to their source regions. The seven instruments of IMPACT will sample the 3D distribution of solar wind plasma electrons and the local vector magnetic field.

PLASTIC is a prime sensor on STEREO for studying coronal/solar-wind and solarwind/heliospheric processes. It measures the distributions of density, velocity, and kinetic temperature (and its anisotropy), solar wind protons $(\mathrm{H})$ and alphas $(\mathrm{He})$, the elemental composition, charge state distribution, kinetic temperature, and velocity of the more abundant solar wind heavy ions $(\mathrm{C}, \mathrm{O}, \mathrm{Ne}, \mathrm{Mg}, \mathrm{Si}, \mathrm{Fe})$, as well as the distribution functions of suprathermal ions (H through Fe). The PLASTIC measurements at two different heliospheric positions will constrain better the relations between variations of the elemental composition (including the FIP effect) in the solar wind and their coronal origin, by having two spatial checkpoints at $1 \mathrm{AU}$ for theoretical time-dependent 3D models of the heliospheric solar wind. The hope is to understand the acceleration of the solar wind, for instance how the slow solar wind originates near coronal streamer boundaries, or how the recurrent ion events originate near corotating interaction regions (CIRs).

\section{Modeling of Eruptive Filaments}

\subsection{MHD Models of Eruptive Filaments}

The trigger of a flare or CME is often the (magnetic) destabilization and subsequent eruption of a filament (called a prominence if seen over the solar limb), which is initially suspended over a highly-sheared neutral line. The destabilization of the filament can be caused either by the kink instability, during a process of increased twisting, or by some other equilibrium-loss process. It can be initiated by continued shearing of the magnetic field, by increasing currents, by converging motion of magnetic footpoints, by buoyancy with subsequent ballooning, or through new magnetic flux emergence. The physical understanding of the origin of a CME has now evolved from sketchy cartoons inspired by observations to full-scale numerical 3D MHD simulations constrained by observed magnetic fields; for recent reviews see, e.g., Forbes (2000), Klimchuk (2001), Zhang and Low (2005), and Roussev and Sokolov (2005). Let us mention a few of the most recent 3D MHD simulations that seem to be most relevant for modeling of STEREO data.

The eruption of a filament or a magnetic flux rope in a gravitationally confined helmet streamer cavity (in the form of cool, dense prominence material) could be initiated after draining of the prominence material. The buoyancy force causes the rise and eruption of the flux rope, pushing aside the helmet streamer field lines (Low, 1996). A time-dependent 3D (ideal) MHD simulation of this CME eruption model was realized by Gibson and Low (1998), and the 3D structure viewed from different (stereoscopic) aspect angles is discussed in Gibson and Low (2000). Recent 3D MHD simulations of the Gibson-Low model of a buoyantly emerging magnetic flux rope were performed by Manchester et al. (2004a). The steady-state coronal field in the MHD model is generated from a prescribed dipole field that progressively is opened up by the solar wind at high latitudes. Then a Gibson-Low type flux rope is inserted inside a closed magnetic loop. To initiate the filament eruption, about $20 \%$ of the balancing mass is removed from the flux rope, which produces an unbalanced pressure that brings the flux rope out of equilibrium. Future models will incorporate self-consistent arcade eruptions, based on the new insight that the magnetic field and shear velocity are not independent (Manchester, 2003; Manchester et al., 2004b). 
Another line of CME initiation models is based on the analytical model of Titov and Démoulin (1999), which contains a flux rope that is suspended in the corona by a balance between magnetic compression and tension forces. In the 2D models, the flux rope with current $I$ has two possible equilibrium positions, provided that the current is not too large: The lower position is stable, while the upper position is unstable. Above a critical current there are no equilibria, and a small outward displacement leads to eruption of the flux rope. In a modified version of the Titov and Démoulin (1999) model developed by Roussev, Sokolov, and Forbes, the flux rope has a poloidal force-free field produced by a (toroidal) ring current and a toroidal force-free field produced by azimuthal currents. An example of such a 3D MHD simulation of an erupting flux rope is shown in Roussev et al. (2003b), with the initial configuration illustrated in Figure 7. A special application of this CME model is illustrated in Roussev et al., 2004 (Figure 8): The fully 3D numerical model of a solar eruption incorporates solar magnetogram data and a loss-of-equilibrium mechanism. The study was inspired by the CME event that took place on May 2, 1998, in NOAA AR 8210 and is one of the SHINE Campaign Events. The CME model has demonstrated that a CMEdriven shock wave can develop close to the $\operatorname{Sun}(\sim 3 R \odot)$, and is sufficiently strong to account for the prompt appearance of high-energy solar protons $(\sim 1 \mathrm{GeV})$ at the Earth. Using this CME model, Sokolov et al. (2004) have carried out a numerical investigation in which they quantified the diffusive acceleration and transport of solar protons at the shock wave from the MHD calculations. The coupled CME-SEP simulation has demonstrated that the theory of diffusive shock acceleration alone can account for the production of $\mathrm{GeV}$ protons during solar eruptions.

A further line of CME initiation models focuses on the kink instability of a twisted flux rope. The force-free coronal loop model by Titov and Démoulin (1999) is found to be

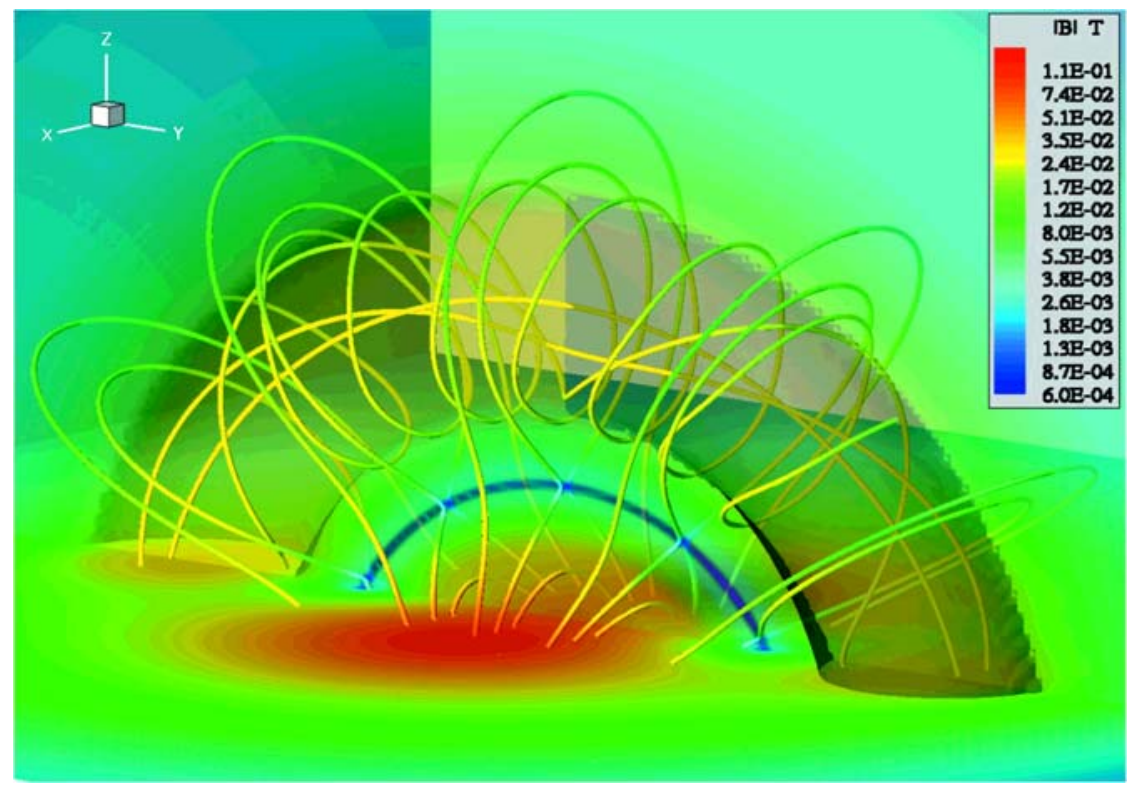

Fig. 7 Initial configuration of the 3D magnetic field of a flux rope prone to loss of equilibrium and subsequent eruption. The solid lines are magnetic field lines, where the false-color code visualizes the magnetic field strength in units of Testa. The surface shaded in gray is an isosurface at $B_{z}=0$ (Roussev et al., 2003b) 


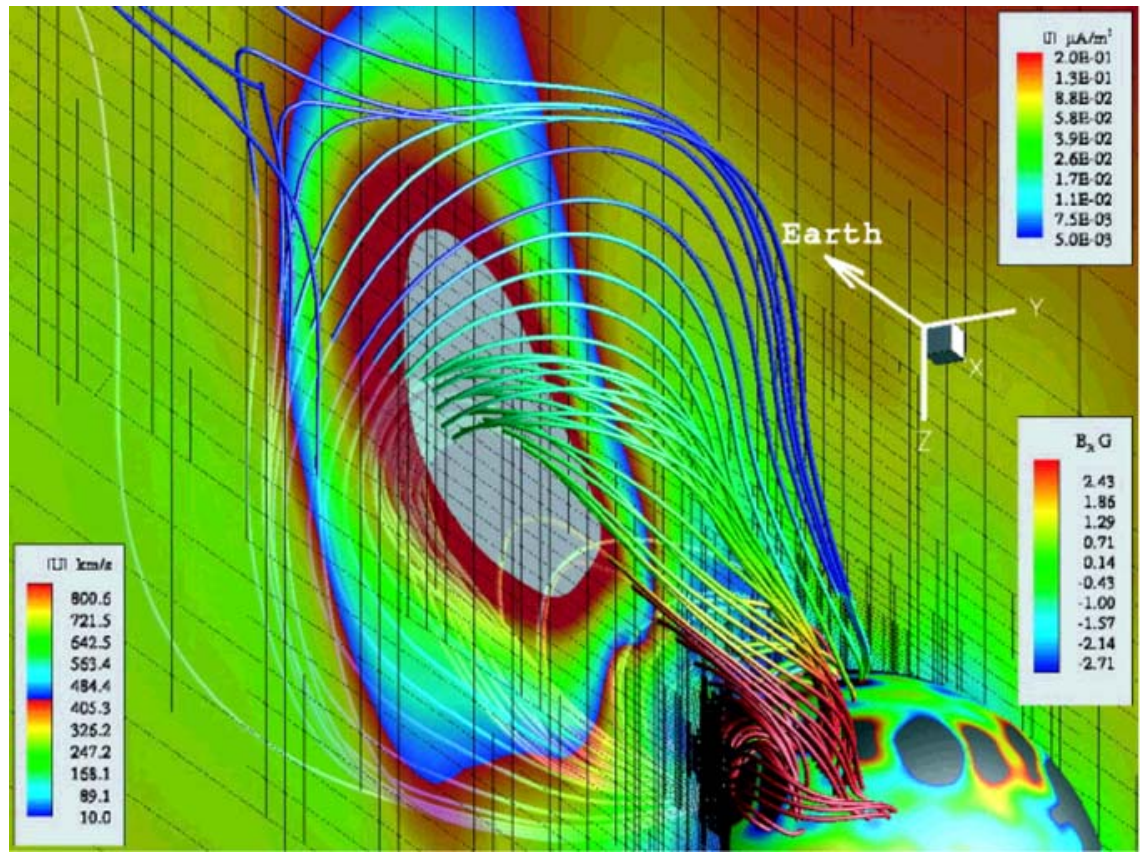

Fig. 8 Three-dimensional view of the modeled CME from May 2, 1998, at $1.1 \mathrm{~h}$ after the initiation (from Roussev et al., 2004). The solid lines are magnetic field lines and the false color shows the magnitude of the current density in units of $\mu \mathrm{A} \mathrm{m}^{-2}$ (see color legend at top right). The magnitude of flow velocity, in units of $\mathrm{km} \mathrm{s}^{-1}$, is shown on a translucent plane (see color legend to the left). Values in excess of $1,000 \mathrm{~km} \mathrm{~s}^{-1}$ are blanked and shown in light grey. The grid-structure on this plane is also shown as the black frame. The inner sphere corresponds to $R=R_{\odot}$. The color shows the distribution of radial magnetic field in units of Gauss (see color legend at bottom right). Regions with field strength greater than $3 \mathrm{G}$ are blanked and appear in grey (Roussev et al., 2004)

unstable with respect to the ideal kink mode, which suggests this instability as a mechanism for the initiation of flares, once the average twist of $\Phi \lesssim 3.5 \pi$ is exceeded (Török and Kliem, 2003; Török et al., 2003; Kliem et al., 2004; Rust and LaBonte, 2005). A particularly fitting simulation of a kinking filament that becomes unstable is shown in Figure 9, where a close ressemblance with EUV images from TRACE $195 \AA$ is demonstrated (Török and Kliem, 2004). The magnetic field decrease with height above the filament is critical whether a confined eruption or a full (unconfined) eruption occurs. Because this model predicts a fairly accurate evolution of the 3D geometry of the kinking filament, a time-dependent 3D reconstruction with two STEREO spacecraft using EUVI images promises very stringent tests of this theoretical model.

More complex CME initiation models involve multiple magnetic flux systems, such as in the magnetic break-out model (Antiochos et al., 1999). In this model, reconnection removes unstressed magnetic flux that overlies the highly stressed core field and this way allows the core field to erupt. The magnetic break-out model involves specific 3D nullpoints and separatrices. A multi-polar configuration was also included in the updated catastrophe model (Lin and Forbes, 2000; Lin and van Ballegooijen, 2005), which contrasts the magnetic breakout model. Such more complex magnetic configurations are difficult to disentangle, but two independent views with the STEREO/EUVI imagers provide a more promising capability to test the 3D magnetic field configuration than previous single-spacecraft observations. 

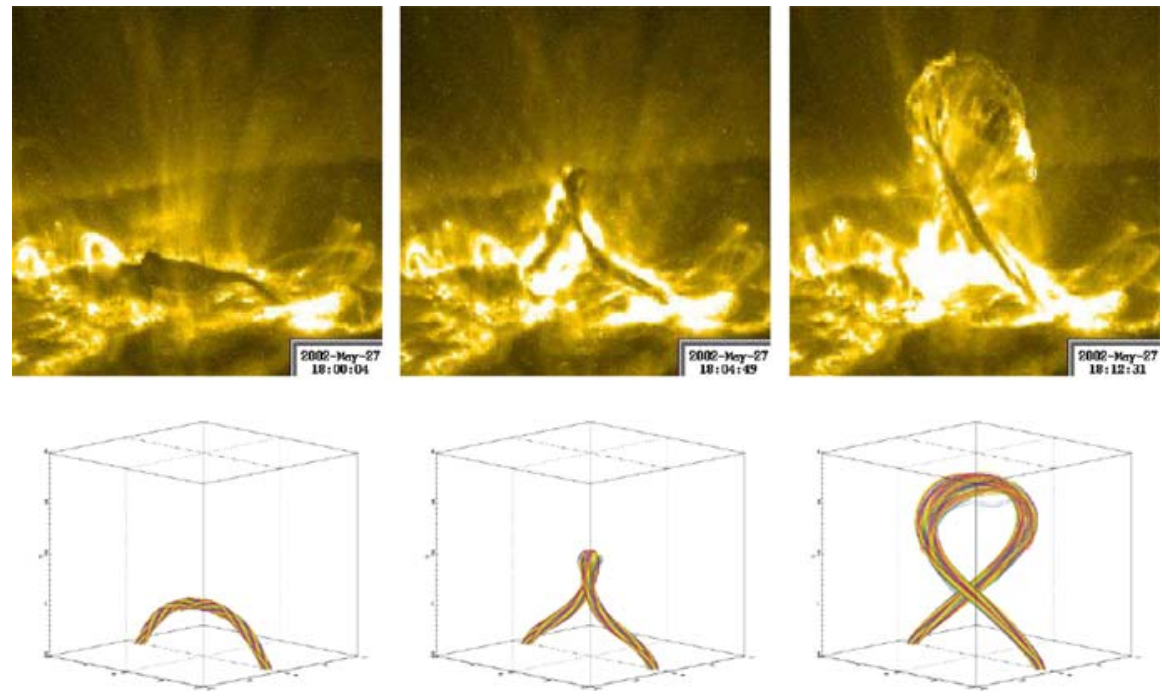

Fig. 9 Top: TRACE $195 \AA$ images of the confined filament eruption on 2002 May 27. The right image shows the filament after it has reached its maximum height. Bottom: magnetic field lines outlining the kink-unstable flux rope reproduced with 3D MHD simulations (Török and Kliem, 2004)

\subsection{Modeling of EUV and White-Light Emission}

While most theoretical models of eruptive filaments are formulated in terms of the 3D magnetic field, quantitative tests with observations require the magnetic field lines to be filled with plasma, so that emission measures and line-of-sight integrated images can be simulated and compared with observed images, e.g., in white-light for SECCHI/COR and HI, or in EUV for SECCHI/EUVI.

Previous comparisons of theoretical models with observed images of eruptive filaments showed evidence for the helical geometry of magnetic flux ropes (Rust and Kumar, 1996; Chen et al., 1997, 2000; Dere et al., 1999; Wood et al., 1999; Ciaravella et al., 2000; Gary and Moore, 2004, Figure 10 here), evident in EUV images in the lower corona as well as in white-light images in the outer corona. There is a strong connection between the magnetic structure of interplanetary magnetic flux ropes (or magnetic clouds) and that of the associated coronal fields at the site of erupting filaments/prominences (Bothmer and Schwenn, 1998; Bothmer, 2003; Cremades and Bothmer, 2004). Some synthetic white-light images have been simulated for a flux rope model by Chen et al. (2000), but an unambiguous test of the 3D geometry requires at least two views with different aspect angles, as SECCHI/COR and HI will provide.

The eruption of a filament or launch of a CME can also be tracked at the base of the solar corona: (1) where a dimming occurs in EUV (Hudson et al., 1998) due to a temporary deficit of evacuated coronal plasma, (2) by detecting the formation of post-eruption arcades in EUV and white-light (Tripathi et al., 2004), or (3) in the form of EIT waves. (Thompson et al., 1999), which concentrically propagate over the entire solar surface, caused by the "pressure implosion" at the epicenter of the erupted filament. The propagation of EIT waves has been theoretically simulated in terms of fast-mode MHD waves (Wang, 2000; Chen et al., 2002; Wu et al., 2001), which helped to reconcile the observed speed of propagating EIT waves with 

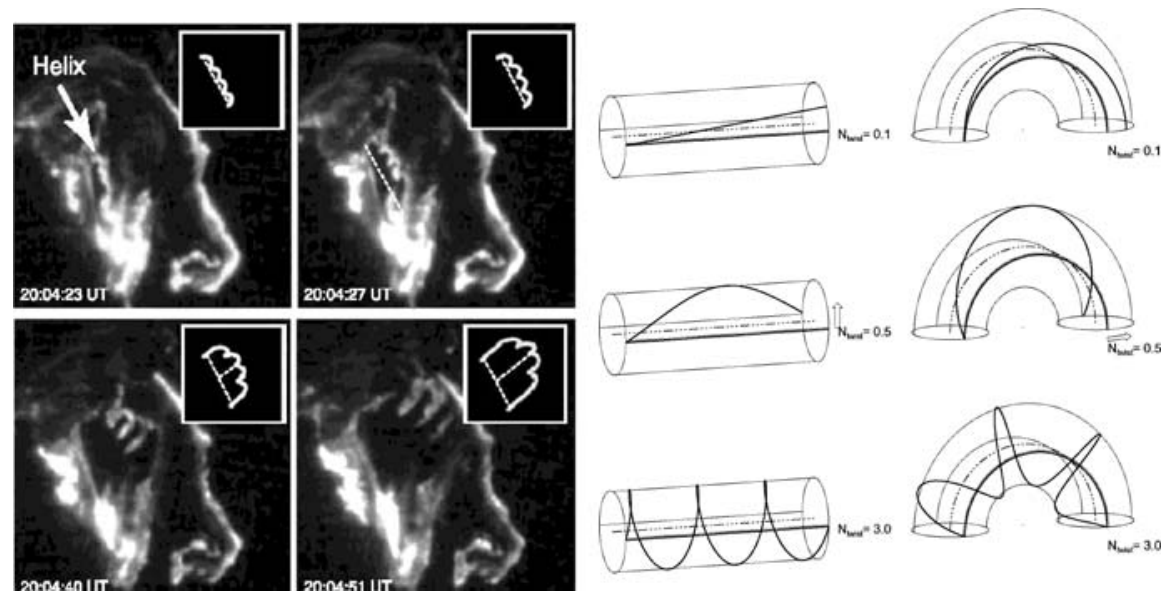

Fig. 10 Left: TRACE $1600 \AA$ images in CIV of the GOES-class X3 flare on 2002-Jul-15, 20:04 UT. The inserts illustrate the geometry of the helical structure, exhibiting 3-4 turns. Note that the helical structure expands, rises, and unwinds during the eruption (Gary and Moore, 2004); Right: Geometrical models of helical fluxtubes with different twists $(0.1,0.5,3.0$ turns $)$, projected onto straight and curved cylinders

the theoretically expected speeds of (fast-mode MHD) magnetoacoustic waves (Figure 11). STEREO/EUVI images enable us to determine the average local density $n_{e}(\mathbf{x})$ and temperature $T_{e}(\mathbf{x})$ (in the range of $T_{e} \approx 0.7-2.7 \mathrm{MK}$ ) of the coronal plasma, while photospheric magnetograms provide input for extrapolation of the coronal magnetic field $B(\mathbf{x})$, and this way the local Alfvén speed $v_{A}(\mathbf{x})$ and sound speed $c_{S}(\mathbf{x})$ can approximately be computed for every location $\mathbf{x}$ in the global corona. This allows us then to predict the (fast-mode MHD) magnetoacoustic wave speed, which in turn can be compared with the observed propagation speed of EIT waves. The SECCHI images will therefore provide powerful constraints for the $3 \mathrm{D}$ propagation of global waves in the corona.

The data search, the objectivity of morphological characterization, and the modeling efficiency can considerably be enhanced by automated detection algorithms, as it has already been faciliated by automated filament detection (Ipson et al., 2005; Zharkova and Schetinin, 2005), by automated detection of EIT waves and dimming (Podladchikova and Berghmans, 2005), by automated CME detection (Robbrecht and Berghmans, 2004), and by automated detection and 3D reconstruction of EUV prominences (Foullon, 2003). In summary, powerful tools for automated feature detection, theoretical 3D models of erupting filaments, and simulations of the corresponding EUV and white-light images have been developed over the last decade, but the feedback algorithms that vary the free parameters in theoretical models and control the forward-fitting to observed images (as we expect from STEREO) are still lacking.

\section{Modeling Coronal Mass Ejections}

\subsection{MHD Simulations of CMEs}

Some key questions of the STEREO mission address the 3D structure and evolution of CMEs from the solar corona to interplanetary space, in particular the physical understanding 

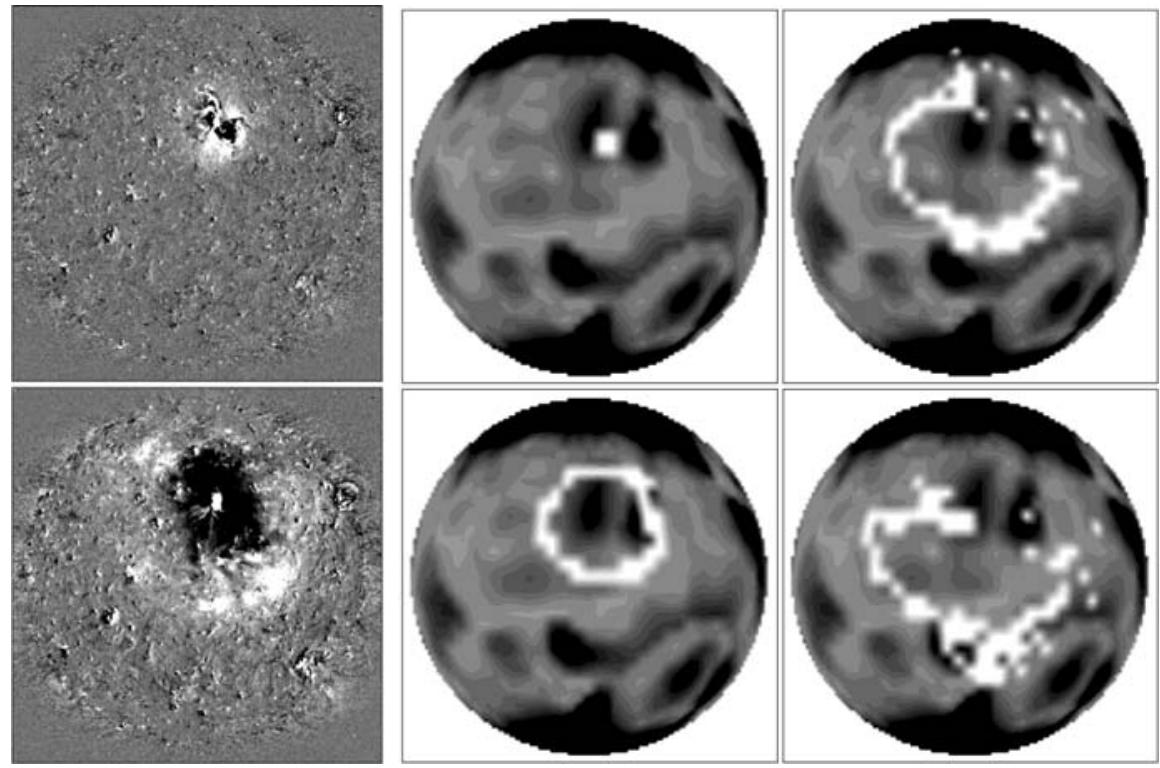

Fig. 11 Left: Two running-difference SoHO/EIT $195 \AA$ images of an EIT wave observed 16 and 30 min after launch of the CME on 1997-May-12, 04:34 UT. Right: Simulation of an EIT wave by a ray-tracing method of fast-mode MHD waves. The color range indicates wave speeds $v>500 \mathrm{~km} \mathrm{~s}^{-1}$ (black) and lower speeds (white). The four simulated images correspond to 2, 15 min (middle column), and 30, 45 min (right column) after launch of the CME (Wang, 2000)

of the forces involved in various acceleration and deceleration phases of propagating CMEs. These questions can only be answered by 3D MHD simulations of CMEs constrained by 3D observations such as those from STEREO. Powerful numerical 3D MHD codes have now become available that are capable of performing the required simulations, such as the coupled MAS/ENLIL code used by the SAIC and NOAA Team (see also Section 3.1), or the BATS-R-US code used by a University of Michigan Team.

As with the ambient solar wind model described in Sections 3.1 and 3.2, SAIC and NOAA/SEC have coupled their models to study the eruption and evolution of CMEs through the corona and into the solar wind. The details of the algorithm used to advance the equations of the SAIC coronal models (MAS) are given elsewhere (Mikić and Linker, 1994; Lionello et al., 1998; Mikić et al., 1999). Briefly, the equations are solved on a spherical $(r, \vartheta, \varphi)$ grid, which permits non-uniform spacing of mesh points in both $r$ and $\vartheta$, thus providing better resolution of narrow structures, such as current sheets. Staggered meshes are employed, which has the effect of preserving $\nabla \cdot \mathbf{B}=0$ to within round-off errors for the duration of the simulation.

Figure 12 illustrates how CME initiation can be modeled self-consistently. The configuration of the solar corona prior to the emergence of the flux rope is summarized in the two left most panels. This type of equilibrium solution has been discussed in more detail by Linker et al. (1999). Contours of the magnetic flux function (fiduciaries of magnetic field lines in two dimensions) are shown by the solid lines and shaded contours (Figure 12, top). The system consists of a single streamer belt displaced by $\approx 10^{\circ}$ below the heliographic equator. The first column shows the state of the corona after the system has reached equilibrium. The second column shows how this configuration is modified by energization of the magnetic field via photospheric shear (Linker and Mikić, 1995). At this point, the system is still in equilibrium. 


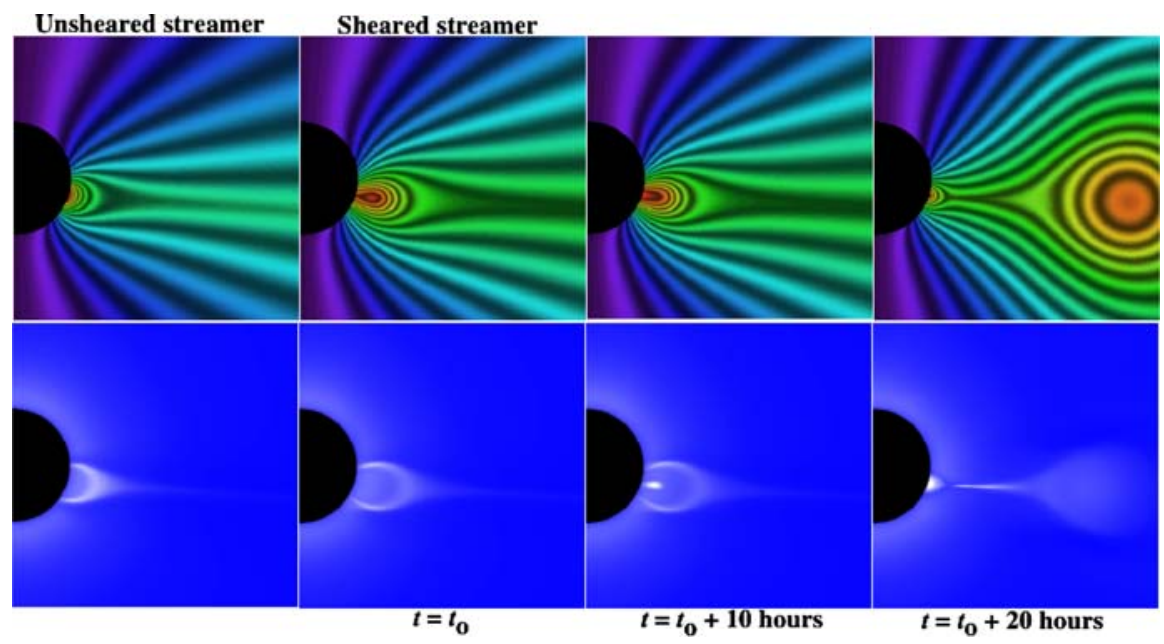

Fig. 12 Evolution of a sheared helmet streamer via flux cancellation. The top panels show contours of the magnetic flux function, which in two dimensions are equivalent to the magnetic field. The bottom panels show the simulated polarized brightness. The four columns summarize: (1) the state of the unsheared corona; (2) the sheared corona; (3) the eruption of the flux rope after $10 \mathrm{~h}$; and (4) the eruption of the flux rope after $20 \mathrm{~h}$, respectively (Riley et al., 2003b)

The polarized brightness $(\mathrm{pB})$ is shown in the Figure 12 bottom panels, constructed by integrating the product of the number density with the scattering function (Billings, 1966) along the line-of-sight (see Section 5.3). The resulting image bears some generic resemblance to SoHO/LASCO white-light images taken near solar minimum (although the model does not reproduce details such as the often observed twisted field lines and bright pre-CME central cores seen in white light images). The remaining panels of Figure 12 summarize the lauch of a flux rope following the cancellation of flux. As can be seen, the origins of the flux rope lie in the closed magnetic field lines embedded within the streamer belt. As the flux rope erupts into the solar corona, overlying field lines, which are still connected back to the Sun at both ends, are brought together under the flux rope. As they reconnect with each other, they contribute both to the flux of the evolving flux rope to the right of the reconnection site and to the re-growth of the streamer belt to the left. Note that the flux rope has developed an elliptical shape, with its major axis approximately in the ecliptic plane. Note also that the reconnection site underneath the erupting flux rope is visible in the simulated $\mathrm{pB}$ image at $t=20 \mathrm{~h}$. This density enhancement was produced by the vertical (i.e., approximately parallel to the solar surface) flow of plasma into the reconnection region and has been observed in white light images (Webb et al., 2003). With regard to the simulated polarized brightness images, we also remark that they bear a strong resemblance to the classic three-part structure of CMEs observed in white light: the bright front, dark cavity, and dense core.

The BATS-R-US solves a set of (ideal) MHD equations using the Block Adaptive Tree Solar Wind Roe-type Upwind Scheme (BATS-R-US) code (Powell et al., 1999; Groth et al., 2000), in combination with the Artificial Wind approximate Riemann (AWR) solver (Sokolov et al., 2002). This is a conservative finite-volume method with shock-capturing total variation diminishing schemes, explicit/implicit time stepping, a block-adaptive mesh refinement scheme, that runs on massively parallel computers. The energy equation is simplified to the kinetic and gravitational terms (neglecting radiative losses, heat conduction, background heating, and dissipative effects due to viscosity and electric resistivity). A series of BATS-R-US 

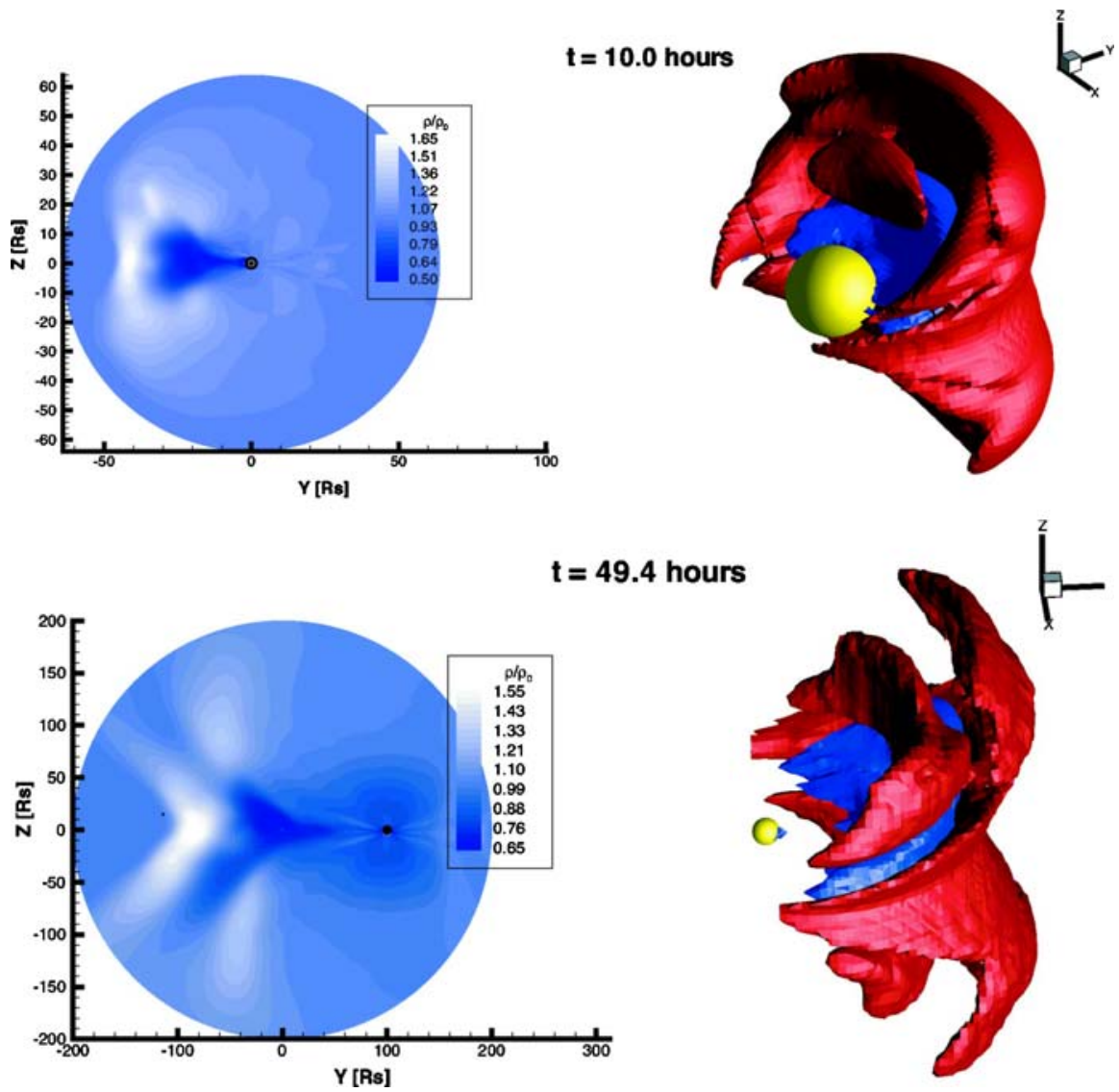

Fig. 13 Top left: Line-of-sight image of a CME simulated with the BATS-R-US code, $10 \mathrm{~h}$ after its launch, as seen from a coronagraph looking at the limb CME with a field of view of $64 R_{\odot}$ centered at the Sun. The black disk, corresponding to $2 R_{\odot}$, shows the occulting disk of the coronagraph. Top right: Two isosurfaces showing the density increase by $30 \%$ (red) and a density decrease of $20 \%$ (blue) over the pre-event density structure, $10 \mathrm{~h}$ after launch of the CME. The yellow sphere is positioned at the Sun and has a radius of $10 R_{\odot}$. Bottom left: Line-of-sight image of the CME, $49.6 \mathrm{~h}$ after launch, with a field-of-view of $200 R_{\odot}$. Bottom right: Similar representation as top right, at $49.6 \mathrm{~h}$ after launch (Lugaz et al., 2005)

runs simulate the launch of a CME by loss of equilibrium of a flux rope anchored on the solar surface (Roussev et al., 2003b), shock formation at a distance of $5 R_{\odot}$ (Roussev et al., 2004), and the evolution of the $\mathrm{CME}$ density structure during propagation out to $100 R_{\odot}$, with simulations of stereoscopic views in white-light (Figure 13) as it will be seen by STEREO/HI-2 (Lugaz et al., 2005).

The ENLIL code, described in the foregoing section on the solar wind (Section 3.2), is a heliospheric code developed by the NOAA Team (Odstrčil et al., 2002) and covers the range from $30 R_{\odot}$ to $1-5 \mathrm{AU}$, using input at the lower boundary from the MAS model that extends from 1 to $30 R_{\odot}$. The heliospheric code is somewhat simpler than the coronal code (which requires to solve the resistive MHD equations), because the ambient solar wind is everywhere super-critical and the ideal MHD equations can be used. This heliospheric code (Odstrčil et al., 1996; Toth 1996; Odstrčil and Pizzo, 1999a,b) solves the ideal MHD equations with an explicit finite-difference scheme, uses an adiabatic constant of $\gamma=5 / 3$ to describe the 

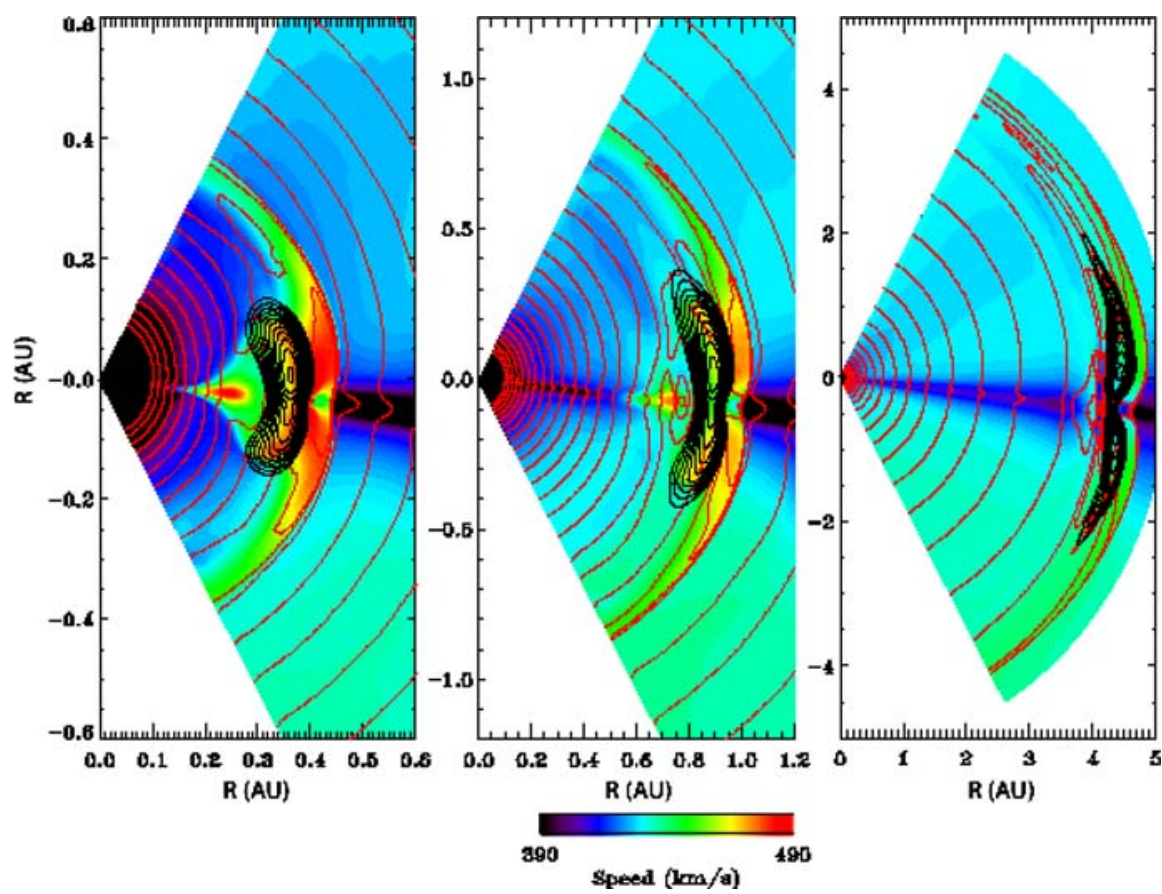

Fig. 14 Evolution of a flux rope through the inner heliosphere. The panels extend $\pm 60^{\circ}$ in latitude and from left to right, extend in heliospheric distance from the Sun to 0.6 AU, 1.2 AU, and $5 \mathrm{AU}$. The contours denote: radial velocity (color); density (red lines); and magnetic field (black lines) (Riley et al., 2003b)

fully-ionized solar wind plasma, and produces accurate shock strengths. This code simulates the distortion of the interplanetary magnetic field by the $3 \mathrm{D}$ propagation of a CME in a structured solar wind (Odstrčil and Pizzo 1999a), the 3D propagation of a CME launched within (Odstrčil and Pizzo, 1999b) and adjacent to a streamer belt (Odstrčil and Pizzo, 1999c) out to $5 \mathrm{AU}$. These runs have shown that the disentangling of merged CME and CIR shocks require multi-spacecraft observations such as STEREO will provide. Simulations of the 12 May 1997 interplanetary coronal mass ejection (ICME) event have enabled us to predict the arrival of the shock and ejecta at Earth (Odstrčil et al., 2004a). Stereoscopic white-light images simulated from these 3D MHD outputs are expected to allow for discrimination between different event scenarios (Odstrčil et al., 2005).

The most comprehensive end-to-end approach of modeling CMEs has been started at the Center for Integrated Space Weather Modeling (CISM), led by Boston University (Principal Investigator: W. J. Hughes). The goal is to simulate the full Sun-to-Earth system by coupling state-of-the-art codes (Luhmann et al., 2004), modeling the solar corona (MAS code), the solar wind (ENLIL code), the magnetosphere, and the upper atmosphere/ionosphere. The propagation of a CME in a coupled coronal (MAS) and heliospheric (ENLIL) MHD code is decribed in Odstrčil et al. (2004b).

Figure 14 summarizes the evolution of a flux rope and its associated disturbances between the Sun and $5 \mathrm{AU}$ at 3 times. The displayed speeds have been restricted to $390-490 \mathrm{~km} \mathrm{~s}^{-1}$ to emphasize flows associated with the disturbance. Note how the ejecta become progressively more distorted with increasing heliocentric distance. $\mathrm{By} \approx 5 \mathrm{AU}$ it has been squeezed so much at low latitudes that it has evolved into two lobes, connected by a thin band of compressed 
field. Surprisingly, much of this distortion can be described by kinematic effects (Riley and Crooker, 2004). More importantly, even under such idealized conditions, the flux rope develops considerable structure, suggesting that interpreting and de-convolving STEREO observations of the same ICME will be a challenge. We also note the presence of outflow associated with post-eruption reconnection underneath the flux rope, which has remained intact within the expansion wave (rarefaction region) behind the flux rope; It has a limited latitudinal extent $\left( \pm 15^{\circ}\right)$ and trails the ejecta by $\approx 35 R_{\odot}$ at $1 \mathrm{AU}$ (middle panel). This aspect of the simulation is discussed in more detail by Riley et al. (2002b).

A comparison of different techniques that fit the magnetic structure of an ICME to forcefree and non-force free flux ropes was performed by Riley et al. (2004). Such end-to-end models of the Sun-to-Earth system are of course extremely important to provide a selfconsistent context for modeling the STEREO multipoint images and multipoint in-situ SEP measurements.

\subsection{Modeling the EUV Emission of CMEs}

The field of view of the SECCHI/EUVI imager extends to about $1.7 R_{\odot}$, so EUV emission of CMEs can only be imaged in the corona during the first few minutes after their launch, while the propagation further out can be tracked in white light with SECCHI/COR (COR1: 1.1-3.0 $R_{\odot}$; COR2: 2-15 $R_{\odot}$ ) and SECCHI/HI (12-318 $\left.R_{\odot}\right)$. The 3D reconstruction of CMEs in EUV can be approached in two different ways: either with forward-fitting using a parameterized 3D density model $n_{e}\left(x, y, z, t, T_{e}\right)$ as a function of space $(x, y, z)$, time $t$, and electron temperature $\left(T_{e}\right)$, or by "tomographic" inversion (e.g., using a back-projection method). The first method can be very computing-intensive if there is a large number of free parameters involved, while the second method suffers from extreme undersampling in the case of two spacecraft only (though an additional third view might be available from the SoHO/EIT telescope).

Although no efficient method has been published yet for the 3D reconstruction of CMEs from stereoscopic EUV images, we expect that some iterative forward-fitting method will be developed in near future that has a feedback between the goodness of the fit and the variation of the free model parameters. Once a geometric density model is specified for a given time $t$, i.e., $n_{e}\left(x, y, z, T_{e}\right)$, the EUV intensity for an optically thin spectral line of wavelength $\lambda_{i j}$ (for transition from atomic energy level $\varepsilon_{j}$ to a lower level $\varepsilon_{i}$ ) for a given line-of-sight in direction $z$ is then

$$
I\left(\lambda_{i j}\right)=A_{X} \int C\left(T_{e}, \lambda_{i j}, n_{e}\right) n_{e} n_{H} d z
$$

where $A_{X}=N(X) / N(H)$ is the abundance factor of element $X$ to hydrogen $H, n_{e}$ the electron density, $n_{H}$ the hydrogen density, and $C\left(T_{e}, \lambda_{i j}, n_{e}\right)$ is the contribution function,

$$
C\left(T_{e}, \lambda_{i j}, n_{e}\right)=\frac{h v_{i j}}{4 \pi} \frac{A_{j i}}{n_{e}} \frac{N_{j}\left(X^{+m}\right)}{N\left(X^{+m}\right)} \frac{N\left(X^{+m}\right)}{N(X)} \quad\left(\mathrm{erg} \mathrm{cm}^{-2} \mathrm{~s}^{-1} \mathrm{ster}^{-1}\right)
$$

with $N_{j}\left(X^{+m}\right)$ the population number of the ionization state $+m$. Since the corona is fully ionized, we can use the so-called coronal approximation by setting the hydrogen density equal to the electron density, $n_{H} \approx n_{e}$ (neglecting the helium electrons), which demonstrates that the (optically thin) EUV emission is essentially proportional to the squared electron density, $I \alpha n_{e}^{2}$, for a given electron temperature. [The electron temperature $T_{e}$ determines the 
ionization equilibrium, collision rates, and EUV contribution function.] For the calculation of the contribution function $C\left(T_{e}, \lambda_{i j}, n_{e}\right)$, there are now codes available in the solar community, e.g., the CHIANTI code (Dere et al., 1997, 2001; Young et al., 1998; Landi et al., 1999; see also URL site in Table 2). The total density function at any point in a CME can then be obtained (at time $t$ ) by integrating over all temperatures, $n_{e}(x, y, z, t)=\int n_{e}\left(x, y, z, t, T_{e}\right) d T$. We need to derive an approximate (timedependent) density model $n_{e}(x, y, z, t)$ from modeling the stereoscopic EUV images, either by forward-fitting or by inversion, in order to faciliate quantitative comparisons and tests of dynamic CME models simulated with 3D MHD codes (Section 5.1).

\subsection{Modeling the White-Light Emission of CMEs}

The SECCHI/COR1, COR2, and HI instruments will track CMEs in white light over a range from $1.1 R_{\odot}$ to $328 R_{\odot}(\approx 1.5 \mathrm{AU})$, so they are the primary imagers for 3D reconstruction of propagating CMEs. 3D reconstruction and visualization of CMEs in white light is mostly led by the Naval Research Laboratory (NRL) and Max Planck Institut für Sonnenforschung $(M P S)$ Teams. The goal is to reconstruct the 3D density distribution $n_{e}(x, y, z, t)$ in the solar K-corona, such as polar plumes, equatorial streamers, CMEs, and the coronal volume inbetween. Information is available in total brightness $(B)$ images as well as in polarized brightness $(p B)$ images. Standard tomographic methods are not suitable for only two projections. Maximum entropy and pixon methods (Puetter, 1995, 1996, 1997; Puetter and Yahil, 1999) are considered as more viable, currently investigated by the NRL Team. Current tests with a pixon code require relatively long computing times, but demonstrate successful reconstructions of simple CME geometries (e.g., cones or semi-shells).

In order to reconstruct the electron density from the image of the K-corona captured by the spacecraft, we have to integrate the Thomson-scattered light from all directions that are incident on the spacecraft. The scattered radiation can be separated into tangentially and radially polarized light (Billings, 1966), where the tangential emission coefficient $I_{t}$ may be written as

$$
I_{t}(\mathbf{r})=\frac{\pi I_{0} \sigma}{2} n_{e}(\mathbf{r}) \Sigma_{A} \quad\left(\text { photons s }^{-1}\right)
$$

and the radial emission coefficient $I_{r}$ may be written as

$$
I_{r}(\mathbf{r})=\frac{\pi I_{0} \sigma}{2} n_{e}(\mathbf{r})\left[\Sigma_{B} \cos ^{2}\left(\chi_{s}\right)+\Sigma_{C}\right] \quad\left(\text { photons s }{ }^{-1}\right) .
$$

$I_{0}$ is the solar intensity at disk center, $R$ is the solar radius, $r$ is the distance of the scattering point from Sun center, $\sigma$ is the Thomson scattering cross section, $\chi_{s}$ is the scattering angle, and $\Sigma_{A}, \Sigma_{B}$, and $\Sigma_{C}$, are functions of $r / R$ which account for the non-zero radius of a limbdarkened Sun (Billings, 1966; Minnaert, 1930; Milne, 1921; Neckel and Labs, 1994). The polarized $(p B)$ and unpolarized brightness $B$ are given by

$$
p B=I_{t}-I_{r}
$$

and

$$
B=2 I_{r}
$$


Recent applications of the proper treatment of Thomson-scattered emission of CMEs observed over a large range of elongations angles are given in Vourlidas and Howard (2006).

Note that there are two important differences to EUV imaging: (1) white light emission is proportional to the total density, while EUV emission is proportional to the squared density, and (2) white light sees the total density summed over all temperatures, while EUV images see only the density in the temperature range of a particular filter. Simulations of white-light images from model 3D density distributions are visualized in Lugaz et al. (2005) and in Pizzo and Biesecker (2004). The latter study demonstrates a robust triangulation method to obtain the centroid location, approximate shape, and velocity of CMEs, using a sequence of stereoscopic white-light images. Some new insight about the 3D configuration of CME shapes is also obtained from a recent data analysis study by Cremades and Bothmer (2004), which shows that CMEs arise in a self-similar manner from pre-existing smallscale loop systems, overlying regions of opposite magnetic polarities, which can be exploited to predict some geometric properties based on the relative orientation of the underlying neutral line in each hemisphere.

Based on the density determination of CMEs from white-light images, the total mass and velocity of a CME can be quantified during propagation, which allows to study the energetic balance between potential, kinetic, and magnetic energy, whose sum is found to be approximately conserved based on LASCO data (Vourlidas et al., 2000). However, the thermal energy generated by cumulative heating during its evolution can add a comparable amount to the energy budget of CMEs (Akmal et al., 2001).

A complementary method of 3D reconstruction of CMEs in white light is the method of 3D polarimetric imaging (Moran and Davila, 2004; Dere et al., 2005). The underlying assumption in this method is that the polarized brightness increases for Thomson scattering with $I_{p} \propto \sin ^{2} \chi$, while the unpolarized brightness decreases with increasing $\sin ^{2} \chi$. This information can be used to distribute the mass $n_{e}(x, y, z)$ along each line-of-sight $z$ in such a way that it matches both the polarized brightness $p B(x, y)$ and unpolarized brightness $B(x, y)$. Although this method can be used for a single white light imager (e.g., as demonstrated for SoHO/LASCO), it promises an even better constrained 3D reconstruction for two stereoscopic spacecraft, and thus will provide a very useful test for alternative reconstruction methods (such as pixon).

Further out in the heliosphere, the 3D density distribution of CMEs can be reconstructed tomographically either from polarized brightness data or from interplanetary scintillation (IPS) data (Jackson and Froehling, 1995; Jackson and Hick, 2002, 2004), as mentioned in Section 3.2 (Figure 6).

\subsection{Modeling Radio Emission of CMEs}

Although there is no radio imaging capability onboard the STEREO spacecraft, we emphasize that ground-based radio imaging can provide a very useful complement for $3 \mathrm{D}$ reconstructions of CMEs. In the CME event of 1998-Apr-20 it was demonstrated for the first time that an expanding CME can be imaged directly at (metric) radio wavelengths, based on the nonthermal synchrotron emission from electrons with energies of $\approx 0.5-5 \mathrm{MeV}$ (Bastian et al., 2001). CMEs might even be imaged in radio wavelengths based on their thermal free-free emission (Gopalswamy and Kundu, 1993; Bastian and Gary, 1997), which would help to constrain their 3D density and temperature distribution. Joint radio imaging (with the Nançay radioheliograph) and SoHO/LASCO observations of a CME indicate also successive magnetic reconnection events at the $\mathrm{CME}$ leading edge that are responsible for multiple injections of electrons into interplanetary space (Pick et al., 1998). 


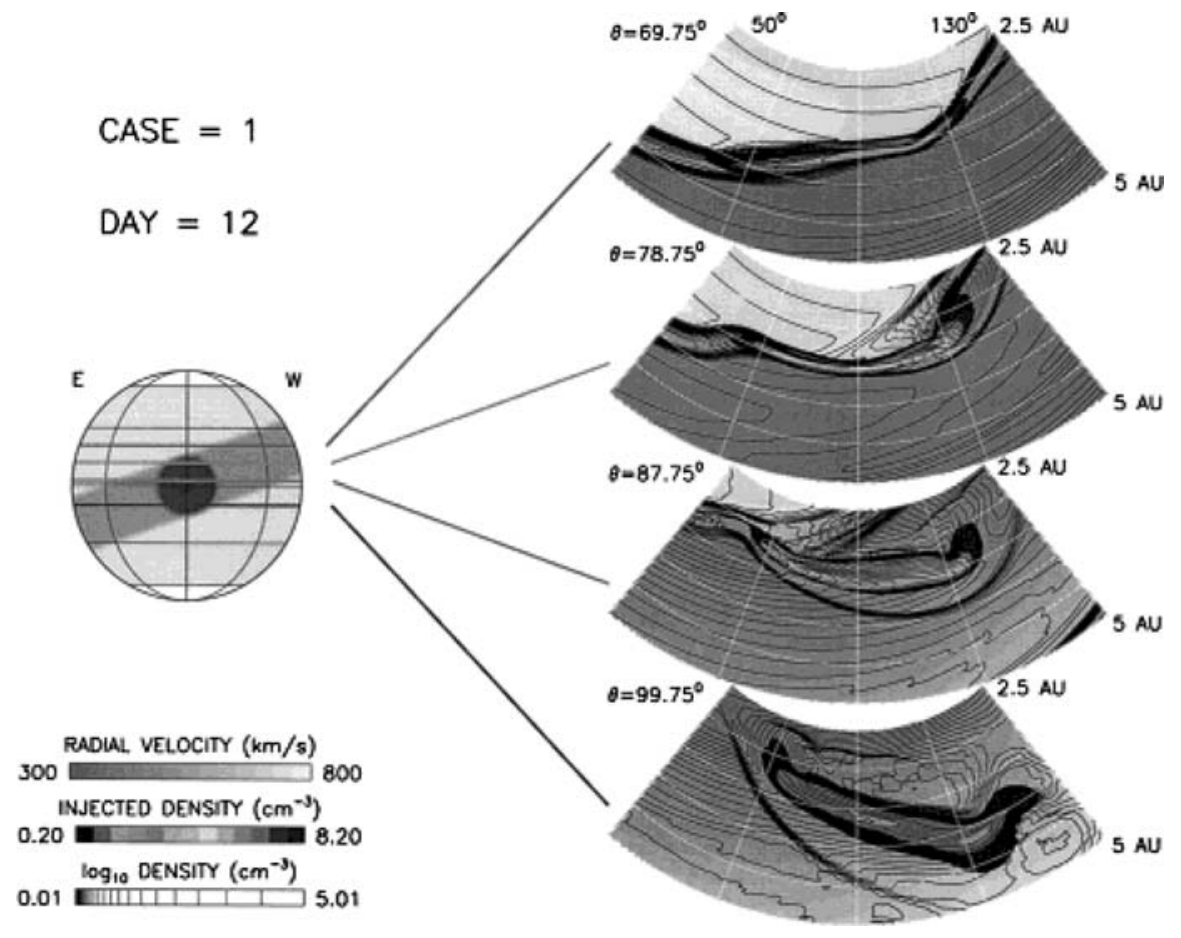

Fig. 15 Numerical MHD simulations of a CME shock wave moving through the ambient solar wind. The CME is injected in the center of the heliospheric current sheet streamer belt (left), which is tilted to the solar axis. The propagating CME is shown at slices in heliographic longitudes and at distance of 2.5-5 AU from the Sun 12 days after launch. The slices are 4 different heliographic latitudes and show how the CME's shape, pressure and speed vary depending on the ambient solar wind conditions (Courtesy of Victor Pizzo)

\section{Modeling Interplanetary Shocks}

\subsection{MHD Modeling of Interplanetary Shocks}

CMEs have typical propagation speeds of $v \approx 300-400 \mathrm{~km} \mathrm{~s}^{-1}$, but fast CMEs have been measured in excess of $v=2000 \mathrm{~km} \mathrm{~s}^{-1}$. The fast solar wind has a typical speed of $v \approx$ $800 \mathrm{~km} \mathrm{~s}^{-1}$. The fast-mode speed dictates whether a fast-mode shock will form, leading to CME driven transient interplanetary shocks. Numerical simulations with HD or MHD codes (e.g., Figure 15), have been able to reproduce the observed speeds and pressure profiles of shocks and CME events out to large distances from the Sun. In such simulations, a pressure pulse is initiated in the lower corona. As the front of a fast CME overtakes the slower solar wind, a strong gradient develops and pressure waves steepen into a forward shock propagating into the ambient wind ahead, and occasionally a reverse shock propagates back through the CME towards the Sun. Numerical simulations of CMEs propagating from the corona (Mikić and Linker, 1994; Linker and Mikić, 1995; Linker et al., 2001) through the heliosphere can be found in Odstrčil et al. (1996, 2002), Odstrčil and Pizzo (1999a, b, c), and Odstrčil et al. (2005). The shock strength as well as the stand-off distance between the shock front and the CME driver gas can vary considerably across the structure, depending where compression or rarefaction occurs between the slow solar wind in the streamer belt and the fast solar wind 
in coronal holes (Odstrčil and Pizzo, 1999b,c). The predicted arrival time of CME shocks at 1 AU depends critically on the modeling of the background solar wind, which controls the shock propagation speed (Odstrčil et al., 2005).

There are a number of complications that can occur, such as the fact that a faster CME can catch up with a slower CME and interact (Gopalswamy et al., 2001). Such interactions form compound streams in the inner heliosphere.

These systems continually evolve further and merge with other CMEs and shocks as they move outward. In the outer heliosphere, beyond $5 \mathrm{AU}$, such structures form Global Merged Interaction Regions (GMIRs), which become so extensive that they encircle the Sun like a distant belt. Such regions block and modulate galactic cosmic rays (i.e., the flux of high-energy particles that continuously streams into the heliosphere). Finally, a forward interplanetary shock wave that passes the Earth's magnetosphere may cause a sudden commencement of a magnetic storm or substorm at the Earth and change the electrical and magnetic connection of the interplanetary magnetic field with the Earth's magnetic field.

\subsection{Detection of Interplanetary Shocks by STEREO}

The kinematic 3D reconstruction of a CME leading edge with SECCHI/COR and HI will provide the true $3 \mathrm{D}$ velocity $\mathbf{v}(\mathbf{r})$ of the propagating CME front, while previous measurements with a single spacecraft (e.g., with SoHO/LASCO) yielded only the velocity component projected in the plane-of-sky, and thus only a lower limit. A large number of CMEs will therefore reveal a higher propagation speed than previously reported values, which may also give a systematic correction from subsonic to supersonic propagation speeds. Triangulation measurements with SECCHI will therefore be an important diagnostic of the true Mach number of interplanetary shocks.

The double-spacecraft configuration of STEREO will also provide situations where a CME shock passes one spacecraft, while the other can observe the CME shock from the side. This provides a unique opportunity to relate the in-situ measurements of shock-accelerated or shock-trapped particles at one spacecraft to the density and velocity diagnostic from the other spacecraft. Specific modeling of such situations has not been published so far, but we anticipate that such data analysis will provide insights into shock acceleration, the primary shock structure, its interactions with corotating streams, interaction regions (CIRs), secondary interplanetary shocks, and transient (solar wind) flows. Detection of radio waves from shockassociated particle beams and energetic particles (SEPs) will be discussed in more detail in the next two Sections (Sections 7, 8).

\section{Modeling of Interplanetary Particle Beams and Radio Emission}

\subsection{Modeling for STEREO/WAVES}

Interplanetary radio bursts provide a rich diagnostic on the acceleration and propagation of energetic particles and shock waves (Figure 16). Radio bursts with plasma frequencies $\lesssim 20 \mathrm{MHz}$ (above the Earth's ionospheric cutoff frequency) can be observed with groundbased radio telescopes. These radio bursts extend only out to about 1-2 solar radii, while all interplanetary radio bursts further out have lower plasma frequencies and require spacebased radio detectors such as STEREO/SWAVES. Previous stereoscopic radio experiments (STEREO-1) with a single spacecraft and a ground-based instrument were able to map out the directivity pattern of type III bursts (Caroubalos and Steinberg, 1974; Caroubalos et al., 


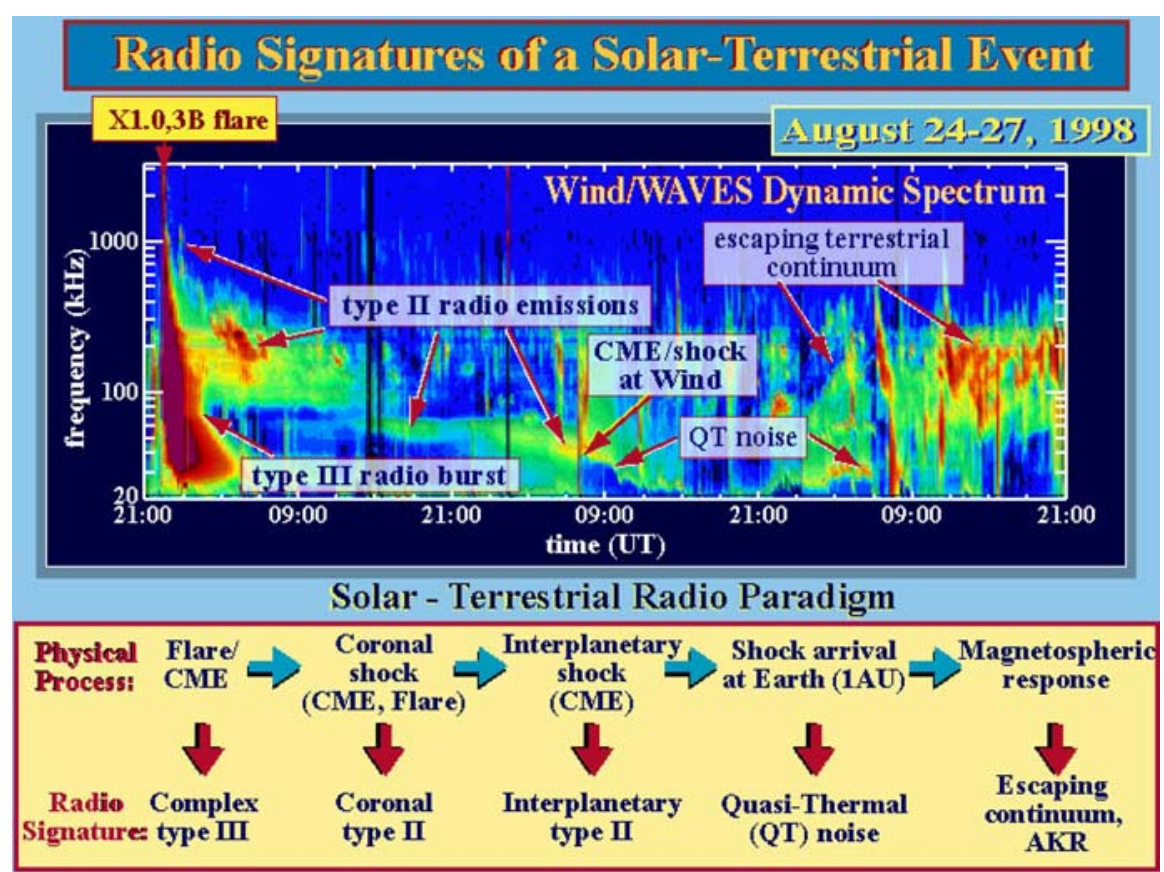

Fig. 16 Overview of physical processes and corresponding radio signatures produced by a flare/CME event. The radio dynamic spectrum is observed by the WIND spacecraft for the 1998 Aug 24-27 geoeffective event (SWAVES website)

1974; Reiner and Stone, 1986, 1988, 1989), while a combination of three spacecraft was able to resolve the 3D trajectory of type III bursts and to demonstrate harmonic emission (Gurnett et al., 1978; Reiner et al., 1998b; Dulk et al., 1985).

The STEREO/WAVES (SWAVES) instruments will have two vantage points in space, and can also be combined with a third viewpoint from ground (at least for frequencies $\lesssim 20 \mathrm{MHz}$ ). SWAVES will be able to triangulate type II and type III radio emission and can observe them remotely as well as in situ together with associated plasma waves, while IMPACT and PLASTIC instruments can detect radio-associated nonthermal particles in situ. The twopoint wave measurements by the two identical SWAVES instruments (combined with the particle detections by IMPACT and PLASTIC) can map out the acceleration efficiency and conversion efficiency into radio waves at two geometrically different parts of a shock, for instance in parallel shock regions (at the CME front) and in perpendicular shock regions (in the flanks of a CME), for large stereoscopic separation angles later in the mission. Previous measurements showed that type II emission upstream of a strong CME-driven interplanetary shock is strongest in quasi-perpendicular shock regions (Bale et al., 1999). The triangulation of the strongest radio type II source as a function of time will track the location of the most efficient particle acceleration and conversion into radio emission within a propagating shock front. The triangulation of multiple radio sources will reveal the detailed shock structure (e.g., foreshock regions). Furthermore, since SWAVES can triangulate the absolute position of plasma emission sources, the plasma frequency and related electron density $n_{e}(\mathbf{r})$ can be determined directly without using heliospheric density models. The triangulated radio source will also yield the direct radial speed $\mathrm{v}(\mathbf{r})$ of the CME-driven shock from the Sun to $1 \mathrm{AU}$, providing real-time predictions of the shock arrival at Earth. 


\subsection{Particle Beams and Radio Type III Emission}

Particle beams, i.e., nonthermal particles with an anisotropic velocity distribution concentrated in a direction parallel to the magnetic field, reveal flare-associated or CME-associated acceleration processes. Flares can produce interplanetary particle beams if the coronal magnetic reconnection site is connected with interplanetary space via open magnetic field lines. Alternatively, interplanetary particle beams might be generated in situ in interplanetary superAlfvénic CME shock waves. So, the localization and tracking of these dual sources of interplanetary particle beams will be a fitting task for the STEREO mission.

Since the plasma in interplanetary space is nearly collisionless, suprathermal and highenergy particles can propagate through interplanetary space and form particle beams (e.g., electron beams or ion beams). The velocity dispersion causes the higher energy electrons to stream ahead of the lower energy electrons, creating a transient bump-in-tail instability. The free (kinetic) beam energy is converted into Langmuir waves via the Landau resonance, and some Langmuir wave energy is converted into radio waves at the fundamental or harmonic local plasma frequency (e.g., McLean and Labrum 1985). Thus, beam-driven type III-like radio bursts are common in interplanetary space. The spatial size of interplanetary radio bursts can be very large, since the extent of the radio source grows with distance from the Sun. A quantitative model of interplanetary type III emission, which incorporates large-angle scattering and reabsorption of fundamental emission amid ambient density fluctuations, called stochastic growth theory, accounts for anomalous harmonic ratios, the exponential decay constant of bursts, burst rise times, arid the directivity of type III emission (Robinson and Cairns, 1998a,b,c), which is suitable for comparisons with SWAVES and IMPACTmeasurements.

\subsection{Shock Waves and Radio Type II Emission}

Classic radio diagnostics of propagating shock fronts are type II bursts, which are characteristic of plasma emission at the fundamental and harmonic plasma frequency generated in coronal and interplanetary shocks, appearing as slowly-drifting pair bands in radio dynamic spectra. Type II bursts are interpreted in terms of shock waves, either CME-driven or blast waves, that accelerate electrons and produce radio emission near the electron plasma frequency $f_{p e}$ and near $2 f_{p e}$ in the upstream region (Wild et al., 1963; Nelson and Melrose, 1985; Bale et al., 1999; Cairns and Kaiser, 2002; Warmuth and Mann, 2005). However, there is no one-to-one correspondence between the existence of shocks and type II bursts. Slowlydrifting type II bursts mark the passage of a shock, but not all shocks produce radio bursts. Furthermore, type II bursts do not outline the entire shock front, but occur only where a shock wave intersects preexisting structures (Stewart, 1984; Reiner and Kaiser, 1999). However, interplanetary type II bursts were all found to be associated with fast CMEs, with shock transit speeds $\gtrsim 500 \mathrm{~km} \mathrm{~s}^{-1}$ (Cane et al., 1987).

Dynamic spectra of both coronal and interplanetary type II bursts routinely show multiple emission bands that appear and disappear, have different frequencies and frequency drift rates, and time varying intensities (e.g., Reiner et al., 1998a; Cane and Erickson, 2005). One goal of the two STEREO/SWAVES instruments is to remotely track type II bursts and interpret the varying frequency fine structures in terms of emission from spatially distinct regions of the shock as they move through the inhomogeneous solar wind. This inversion requires detailed theoretical modelling of type II emission. Recent MHD simulations of CME shocks show also that a single flare/CME event can generate coronal disturbances observed as two separate type II radio bursts (Odstrčil and Karlický, 2000). 


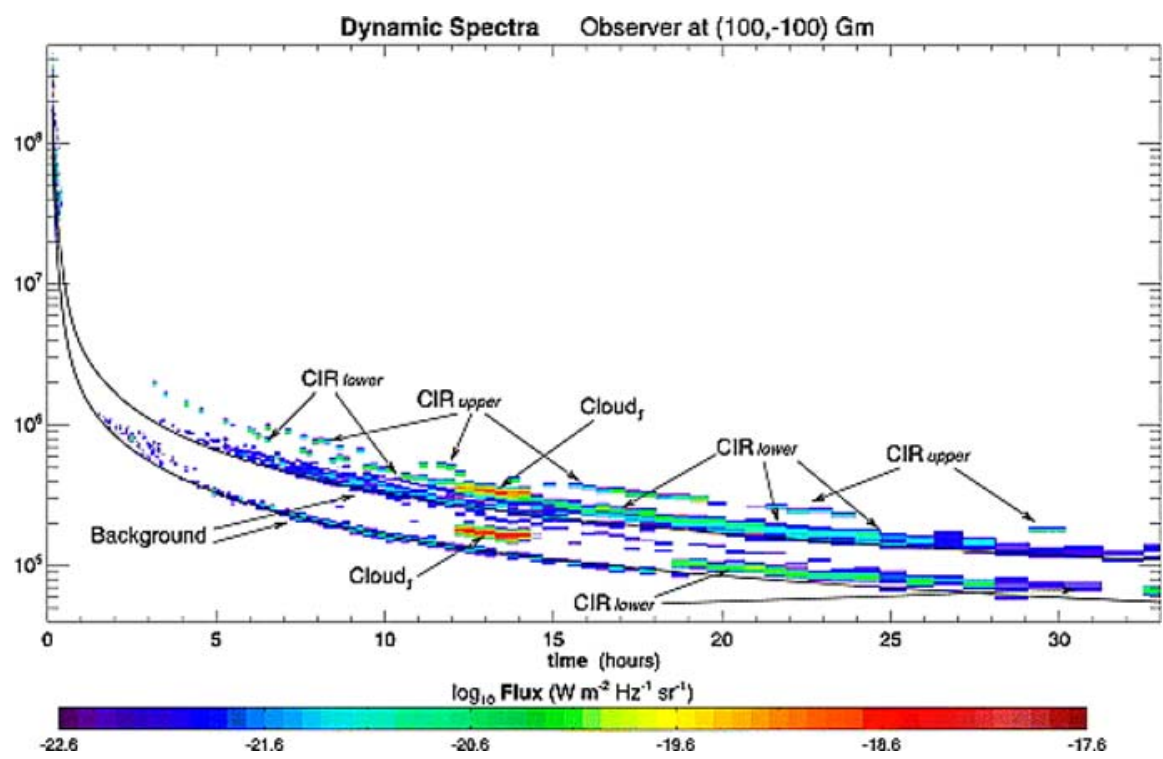

Fig. 17 Dynamic spectrum of a modeled type II burst. The two solid curves are the fundamental and harmonic frequency drift rate of the shock's leading edge. The structures responsible for various spectral features are indicated (Knock and Cairns, 2005)

A semi-quantitative theory exists for type II bursts (Knock et al., 2001, 2003a,b; Knock and Cairns, 2005), which combines (i) "magnetic mirror" reflection and acceleration of upstream electrons incident on the shock, using magnetic moment conservation in the de HoffmanTeller frame, (ii) formation of foreshock electron beams by "time-of-flight" effects, using Liouville's theorem, (iii) estimation of the net energy flow Langmuir waves driven by the electron beams, using quasilinear relaxation and stochastic growth theory, (iv) conversion of Langmuir energy into radiation near $f_{p e}$ and $2 f_{p e}$, using nonlinear Langmuir wave processes with known conversion efficiencies, with shock propagation through an inhomogeneous solar wind. Figure 17 shows the dynamic spectrum predicted for a shock moving through an MHD Parker-model solar wind with two corotating interaction regions (CIRs), two magnetic clouds (e.g., associated with CMEs), and random small-scale inhomogeneities in plasma quantities like density, flow speed, and vector magnetic field (Knock and Cairns, 2005). Features associated with the shock's interactions with specific CIRs and clouds are identified (cf., Reiner and Kaiser 1999; Gopalswamy et al., 2001), while the smaller time scale variations are due to the random solar wind turbulence leading to enhanced or decreased emission from localized regions of the shock. Moreover, predictions for multiple observers show considerable differences interpretable in terms of proximity and frequency-blocking effects, directly relevant to future interpretations of STEREO data. The type II burst model of Knock and Cairns (2005) reproduces a number of observed features that can be used for more detailed diagnostic of the underlying shocks. For instance, the intensity of type II bursts is strongly diminished near a peak in the heliospheric Alfvén speed profile. Other features observed in dynamic spectra of type II bursts, such as multiple-lane effects, variations in the frequency-time drift rate $d v / d t$, onsets and turn-offs of emission, narrowband and broadband emission, can be reproduced with this type II model by inserting local structures in the coronal or interplanetary plasma. 
It is envisaged that the microscopic physics of this and other theories will be tested and improved using future IMPACT and SWAVES data, extended to include macroscopic shock and solar wind models and directivity effects, and used to interpret STEREO white light and radio data in terms of CMEs, shocks, and other interplanetary structures. For a full understanding of the link between CMEs and type II bursts, knowledge of strong interplanetary shocks, the macroscopic and microscopic structure of CME-driven shocks, the generation mechanism of radio emission, and the radiation beaming pattern are required.

\section{Modeling of Solar Energetic Particles (SEPs)}

Solar energetic particle (SEP) events refer to accelerated high-energy particles detected in the heliosphere. Some originate in solar flares, while others are accelerated in transient interplanetary shocks, as they are produced by fast CMEs. The acceleration mechanisms can be DC electric fields, stochastic wave-particle interactions, or shock acceleration mechanisms. Solar energetic particle events are classified into two types, gradual and impulsive SEP events, depending on their energy versus time profile. Gradual SEP events occur with a rate of $\approx 10$ /year during the maximum of the solar cycle, each one can last several days, and they are likely to be accelerated directly in interplanetary shocks rather than by flares in the corona. Impulsive SEP events occur more frequently, with a rate of $\approx 100 /$ year during the maximum of the solar cycle, they last only a few hours, and they are much weaker than gradual SEP events. Since they originate along magnetic field lines connected to coronal flare sites, their acceleration could be governed by the same magnetic reconnection process that governs the associated flare. So, charged particles can be used to trace the interplanetary field topology (Kahler, 1997).

Because the ${ }^{3} \mathrm{He} /{ }^{4} \mathrm{He}$ ratio of some SEPs is much higher than in the normal solar wind, they are also called ${ }^{3} \mathrm{He}$-rich events. Interplanetary particles can also be accelerated in the electric fields that are generated at corotating interaction regions (CIR) between high-speed and low-speed streams. The location where acceleration of interplanetary particles takes place can approximately be determined from the velocity dispersion (i.e., time-of-flight effects), $t_{\text {prop }}=L / \mathrm{v}$, of particles arriving at Earth. Multi-spacecraft observations help us to map the spatial distributions of the accelerated particles that flow out into the heliosphere from the evolving CME shock or those that remain trapped behind it (Reames, 1997). Particularly advantageous opportunities are in-situ particle observations in CME fronts that are observed in Earth-STEREO-Sun quadrature configuration (Figure 18), i.e., when the CME is observed from the side (rather than head-on as with SoHO previously). Such quadrature observations should reveal the shock profile more clearly than at other viewing angles.

\subsection{Theoretical Modeling of SEP Acceleration}

The most recent theoretical modeling of SEP acceleration includes coupled hydromagnetic wave excitation and ion acceleration in an evolving coronal/interplanetary shock (Lee, 2005), the injection problem at a CME-driven shock (Zank and Li, 2004), or SEP acceleration in solar wind compression regions associated with CIRs (Giacalone et al., 2002). The acceleration of solar energetic particles (SEPs) at an evolving coronal/interplanetary CME-driven shock is the most promising theory for the origin of SEPs observed in the large gradual events associated with CMEs (Lee, 2005). This calculation includes the essential features of the 


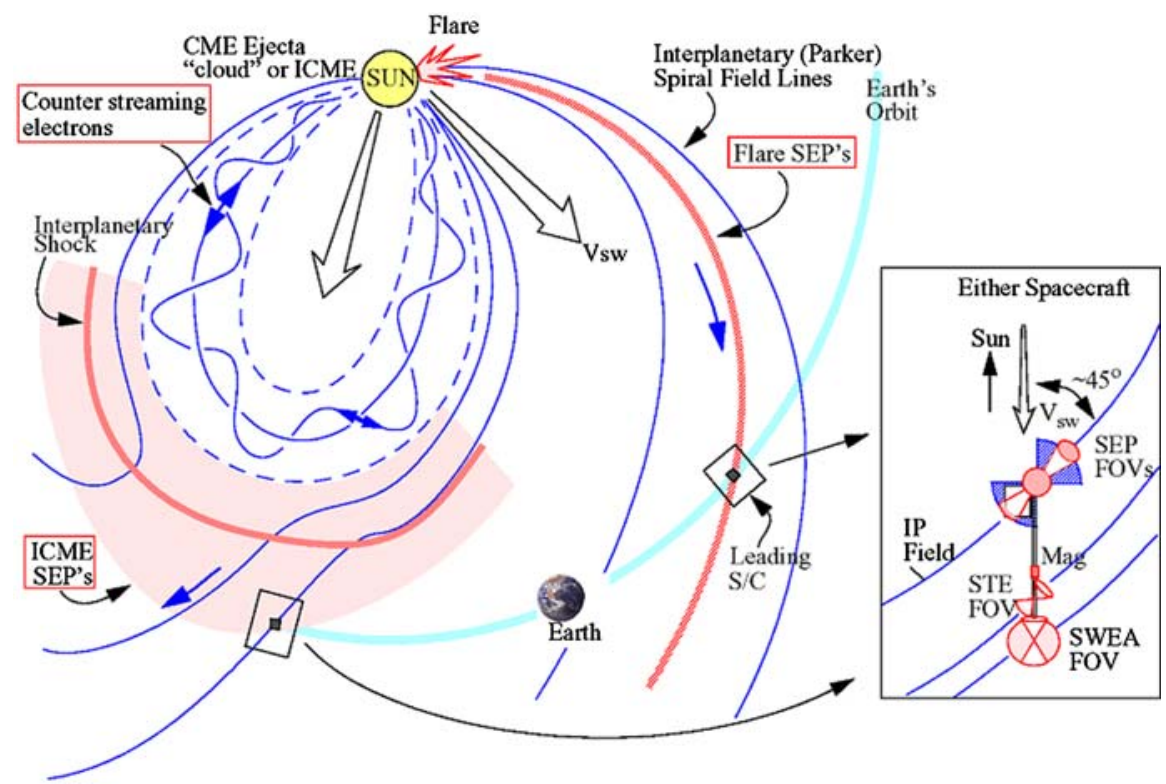

Fig. 18 Possible STEREO/IMPACT observations of SEPs at two magnetically disconnected locations in the heliosphere: STEREO-A is located on an open magnetic field line that is connected to the coronal flare region and will probe flare-accelerated particles, while STEREO-B probes SEPs in-situ in a CME-driven shock in interplanetary space at 1 AU (IMPACT website)

process: diffusive shock acceleration, proton-excited waves upstream of the shock, and escape of particles upstream of the shock by magnetic focusing. The wave spectra and particle distributions predicted are in general agreement with observations but improvement is needed including the form of the excited wave spectrum, which affects ion fractionation and the form of the high-energy cutoff, and a more general velocity distribution for the injected seed population. The seed populations for quasi-perpendicular and quasi-parallel shocks are subjects of current debates. One thought is that quasi-parallel shocks generally draw their seeds from solar-wind suprathermals, while quasi-perpendicular shocks-requiring a higher initial speed for effective injection - preferentially accelerate seed particles from flares. These different origins of seed populations can explain the observed differences in the composition of high-energy SEPs (Tylka et al., 2005).

Numerical modeling of SEP acceleration is now approached by combining MHD fluid codes with kinetic codes, to obtain a self-consistent description of CME shocks and SEP acceleration. In a recent study SEP particles are accelerated in a CME-driven shock at $5 R_{\odot}$ when the shock exceeds a fast-mode Mach number of $\lesssim 4$, producing solar energetic protons with energies below $10 \mathrm{GeV}$, for which a cutoff energy of $\approx 10 \mathrm{GeV}$ would be predicted by diffusive shock acceleration (Sokolov et al., 2004; Roussev et al., 2004).

\subsection{Modeling of SEP for STEREO/IMPACT}

STEREO/IMPACT will sample the 3D distributions of SEP ions and electrons, as well as the local magnetic field (Figure 18). SEP modeling with specific relevance for IMPACT is described in Ng et al. $(1999,2003)$. This line of SEP modeling focuses separately on SEP 

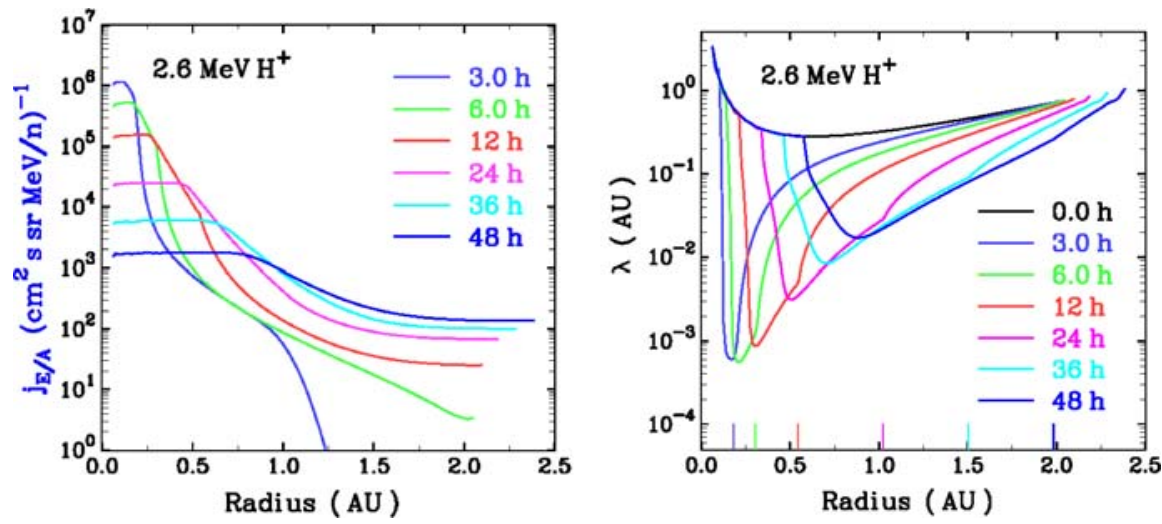

Fig. 19 Coupled evolution of $2.6 \mathrm{MeV}$ proton intensity (left) and mean free path (right) versus radius (Ng et al., 2003)
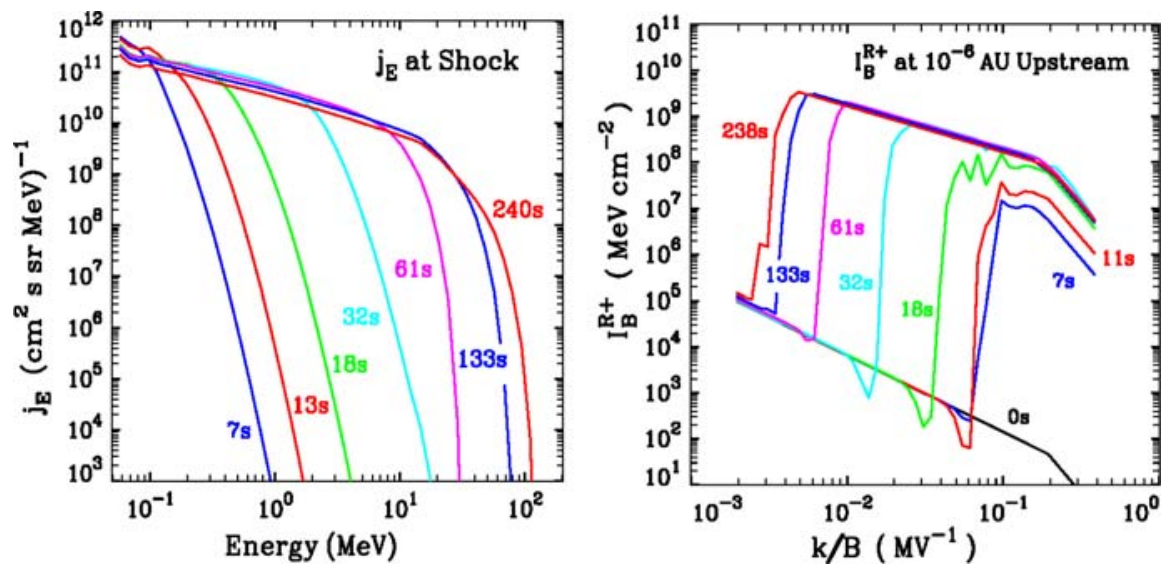

Fig. 20 Proton acceleration (left) and Alfvén wave growth (right) upstream of a moving shock at $\sim 3.7$ solar radii (Courtesy of Chee $\mathrm{Ng}$ and Don Reames)

transport over several AU and their extension to fast acceleration by a coronal shock on fine time and spatial scales. Both efforts study the coupled nonlinear evolution of SEPs and Alfvén waves in inhomogeneous plasma and magnetic field, featuring self-consistent quasilinear wave-particle interaction with full pitch-angle dependence. Both models include focusing, convection, adiabatic deceleration, and scattering (by Alfvén waves) for SEPs, and wave transport and amplification (by SEPs) for the Alfvén waves. The acceleration model treats, in addition, first-order Fermi acceleration and wave transmission/reflection at the shock. The results reveal that, contrary to common assumption, wave amplification strongly impacts SEP acceleration and transport. This transport model predicts the self-throttling of proton transport through wave excitation ( $\mathrm{Ng}$ et al., 2003), as shown by the evolution of the radial profiles of SEP intensity $j_{E}$ and mean free path $\lambda$ (Figure 19). Wave growth also explains the observed complex time variations of SEP elemental abundances (Tylka et al., 1999). The shock acceleration model predicts proton intensity and Alfvén wave spectra evolving in tandem upstream of a $1800 \mathrm{~km} / \mathrm{s}$ shock traveling from 3.7 to 4.3 solar radii (Figure 20). 
Acceleration of $1 \mathrm{MeV}(70 \mathrm{MeV})$ protons "ignites" at $18 \mathrm{~s} \mathrm{(130} \mathrm{s),} \mathrm{when} \mathrm{wave} \mathrm{growth} \mathrm{drives}$ the respective $\lambda$ down from $0.5 \mathrm{AU}(1 \mathrm{AU})$ to below $10^{-4} \mathrm{AU}$. Future work will attempt to integrate the shock acceleration and interplanetary transport models and to generalize it so that it can accept arbitrary input of plasma and shock parameters from other CME and shock models.

Attempts are being made to add SEPs to the Sun-to-Earth end-to-end MHD models at CCMC, CISM, and University of Michigan, which simulate SEP acceleration in realistic CME environments (e.g., Roussev et al., 2004). The STEREO multipoint measurements and multiple viewpoints of the SEP sources will be combined with the models to answer outstanding questions like the relative contribution of flare versus IP shock-generated SEPs in major events. Both the Michigan group and the CISM group are attempting these end-toend system models, and CCMC has the role of a model component provider to STEREO and the largr community.

\section{Modeling of Geoeffective Events and Space Weather}

A key requirement in evaluating geoeffective events and space weather is the determination of CME trajectories towards Earth, with the goal to establish magnetic connectivity and to predict the timing and impact of CME-induced geomagnetic disturbances. While previous single-spacecraft observations (e.g., with SoHO/LASCO) have difficulty in reconstructing the directionality of CMEs, in particular for frontside halo CMEs, the dual vantage point of the two STEREO spacecraft will provide less ambiguous directionality measurements and better arrival forecasts (in real time) from the true 3D vector $\mathbf{r}(t)$ and velocity $\mathbf{v}(t)$ reconstructions by the SECCHI/HI imagers. Once the Sun-Earth connectivity of the CME path is established, we further want to know whether the CME hits the Earth directly, grazes it, or misses it, what the longitudinal extent and cross-section of a CME is, and what the southward magnetic field component $B_{z}$ is (which determines the geoeffectiveness).

Current modeling efforts of space weather are coordinated by Dave Webb (see chapter on Space Weather and Beacon mode) and by Jim Klimchuk at NRL. MHD Modeling for the ESA Space Weather Initiative is coordinated by David Berghmans. An effort to model the geoeffectiveness of CMEs is planned by the $3 \mathrm{D}$ reconstruction group led by Volker Bothmer. Modeling of the magnetic field that connects the subphotospheric domain with the coronal magnetic field during CME initiation is also addressed by the Solar Multidisciplinary University Research Initiative (SOLAR/MURI) at the University of California, Berkeley (UCB). Particular efforts to model space weather by end-to-end simulations of CMEs and SEPs are ongoing at the Center for Integrated Space Weather Modeling (CISM) at the University of California at Berkeley (UCB), and at the Center for Space Environment Modeling (CSEM) at the University of Michigan, which we partly described in Section 3.2 on heliospheric solar wind models. Their Space Weather Modeling Framework (SWMF) aims to come up with a self-consistent framework of models that starts from the CME initiation in the solar corona, follows the CME propagation and SEP acceleration through interplanetary space, and predicts the consequences in the Earth's magnetosphere. Part of their space weather modeling includes also predictions of fluxes and arrival times of high-energy protons at spacecraft locations, which produce a real radiation hazards for manned and unmanned spacecraft. More information of the activities of various groups that perform space weather modeling relevant for the STEREO mission can also be found from the URLs given in Table 2. 
Table 2 Acronyms and URLs of webpages relevant to modeling of STEREO data

\begin{tabular}{|c|c|}
\hline Acronym & Full Name $\mapsto$ Website URL \\
\hline CACTUS & $\begin{array}{l}\text { Computer Aided CME Tracking } \\
\quad \mapsto \text { http://sidc.oma.be/cactus/ }\end{array}$ \\
\hline CCMC & $\begin{array}{l}\text { The Community Coordinated Modeling Center } \\
\quad \mapsto \text { http://ccmc.gsfc.nasa.gov/ }\end{array}$ \\
\hline CHIANTI & $\begin{array}{l}\text { Atomic Database for Spectroscopic Diagnostics of Astrophysical Plasmas } \\
\quad \mapsto h t t p: / / w w w s o l a r . n r l . n a v y . m i l / c h i a n t i . h t m l /\end{array}$ \\
\hline CISM & $\begin{array}{l}\text { Center for Integrated Space Weather Modeling } \\
\mapsto h t t p: / / w w w . b u . e d u / c i s m /\end{array}$ \\
\hline CSEM & $\begin{array}{l}\text { Center for Space Environment Modeling } \\
\mapsto \text { http://csem.engin.umich.edu/ }\end{array}$ \\
\hline IMPACT & $\begin{array}{l}\text { In-situ Measurements of Particles and CME Transients } \\
\quad \mapsto \text { http://sprg.ssl.berkeley.edu/impact/ }\end{array}$ \\
\hline PLASTIC & $\begin{array}{l}\text { PLastic And Supra Thermal Ion Composition investigation } \\
\quad \mapsto \text { http://stereo.sr.unh.edu/ }\end{array}$ \\
\hline SECCHI & $\begin{array}{l}\text { Sun Earth Connection Coronal and Heliospheric Investigation } \\
\quad \mapsto \text { http://stereo.nrl.navy.mil/ }\end{array}$ \\
\hline SECCHI/MPS & $\begin{array}{l}\text { The SECCHI website at Max Planck Institut für Sonnenforschung } \\
\quad \mapsto \text { http://star.rnpae.gwdg.de/secchi/ }\end{array}$ \\
\hline SMEI/UCSD & $\begin{array}{l}\text { Solar Mass Ejection Imager, University California San Diego } \\
\quad \mapsto \text { http://cassfos02. ucsd.edu/solar/ }\end{array}$ \\
\hline $\mathrm{SOHO}$ & $\begin{array}{l}\text { SOlar and Heliospheric Observatory } \\
\quad \mapsto \text { http://sohowww.nascom.nasa.gov/ }\end{array}$ \\
\hline SOLAR-B & $\begin{array}{l}\text { SOLAR-B mission website } \\
\quad \mapsto h t t p: / / w w w . n a s a . g o v / \text { mission-pages/solar-b/ }\end{array}$ \\
\hline SOLAR/MURI & $\begin{array}{l}\text { Solar Multidisciplinary University Research Initiative at UCB } \\
\quad \mapsto \text { http://solarmuri.ssl.berkeley.edu/ }\end{array}$ \\
\hline STEREO & $\begin{array}{l}\text { The Solar TErrestrial RElations Observatory (STEREO) } \\
\quad \mapsto \text { http://stereo.gsfc.nasa.gov/ }\end{array}$ \\
\hline STEREO/SW & $\begin{array}{l}\text { The STEREO Space Weather Group } \\
\quad \mapsto \text { http://stereo.nrl.navy.mil/swx/swindex.html }\end{array}$ \\
\hline SWAVES & $\begin{array}{l}\text { The STEREO Waves Instrument } \\
\quad \mapsto h t t p: / / w w w \text {-lep.gsfc.nasa.gov/swaves/swaves.html }\end{array}$ \\
\hline TRACE & $\begin{array}{l}\text { Transition Region And Coronal Explorer } \\
\quad \mapsto \text { http://sunland.gsfc.nasa.gov/smex/trace/ }\end{array}$ \\
\hline
\end{tabular}

\section{Conclusive Remarks}

In this review we described some theoretical tools that already exist or are being prepared by groups that are committed to the STEREO mission and space weather effort in general, which mostly includes models that try to reproduce transient events in the solar wind and the evolution of coronal mass ejections, but it should not be considered as a complete and exhaustive compilation of relevant theoretical models. There exist a number of alternative solar wind models that explore the physical processes driving the solar wind that are not covered here. Since new observations always challenge existing theories and require new approaches of data modeling we hope that the STEREO mission, once it is launched and produces science data, will stimulate the development of new models and discriminatory tests by data fitting in the future. The anticipated STEREO data base will be an extremely rich database and enable us to model the heliospheric magnetic field and 
propagation of CMEs in unprecedented detail, constrained by true 3D information from stereoscopic vantage points, yielding the 3D kinematics of MHD processes as well as kinetic processes associated with the solar wind, CMEs, and particles accelerated in interplanetary shocks. Since the STEREO mission is our first extensive multi-spacecraft 3D exploration of our heliosphere, its importance might be compared with the first determination of the true 3D geometry of our Earth globe by Thales of Milet and Pythagoras around $600 \mathrm{BC}$.

Acknowledgements We gratefully acknowledge the useful and complementary comments of the two anonymous referees, which helped to improve the readability and accuracy of this paper. Part of the work was supported by NASA contract N00173-01-C-6001 and N00173-02-C-2035 to NRL (SECCHI project).

\section{References}

A. Akmal, J.C. Raymond, A. Vourlidas, B. Thompson, A. Ciaravella, Y. Ko, et al., ApJ 553, 922 (2001)

D. Alexander, G.A. Gary, B.J. Thompson, in Three-Dimensional Structure of Solar Active Regions, ed. by C. Alissandrakis, B. Schmieder, Proc. 2nd Advances in Solar Physics Euroconference, vol. 155 (Astronomical Society of the Pacific (ASP), San Francisco, ASP Conference Series, 1998), p. 100

M.D. Altschuler, G. Newkirk Jr., Solar Phys. 9, 131 (1969)

M.D. Altschuler, R.H. Levine, M. Stix, J. Harvey, Solar Phys. 51, 345 (1977)

M.D. Altschuler, in Image Reconstruction from Projections, ed. by G.T. Herman (Springer, Berlin, 1979), p. 105

S.K. Antiochos, C.R. DeVore, J.A. Klimchuk, ApJ 5(10), 485 (1999)

C.N. Arge, V.J. Pizzo, JGR 105/A5, 10, 465 (2000)

M.J. Aschwanden, T.S. Bastian, ApJ 426, 425 (1994a)

M.J. Aschwanden, T.S. Bastian, ApJ 426, 434 (1994b)

M.J. Aschwanden, J. Lim, D.K. Gary, J.A. Klimchuk, ApJ 454, 512 (1995)

M.J. Aschwanden, Lecture Notes in Phys. 444, 13 (1995)

M.J. Aschwanden, J.S. Newmark, J.P. Delaboudiniere, W.M. Neupert, J.A. Klimchuk, G.A. Gary, et al., ApJ $\mathbf{5 1 5}, 842$ (1999)

M.J. Aschwanden, D. Alexander, N. Hurlburt, J.S. Newmark, W.M. Neupert, J.A. Klimchuk, et al., ApJ 531, 1129 (2000)

M.J. Aschwanden, Physics of the Solar Corona - An Introduction (Praxis Publishing Ltd., Chichester, UK, and Springer, New York, 2004, ISBN 3-540-22321-5), p. 842

M.J. Aschwanden, D. Alexander, M. DeRosa, in Solar and Space Weather Radiophysics, ed. by D.E. Gary, C.O. Keller. Astrophysics and Space Science Library, vol. 314 (Kluwer Academic Publishers, Dordrecht, The Netherlands, 2004), p. 243

M.J. Aschwanden, Solar Phys. 228, 341 (2005)

S.D. Bale, M.J. Reiner, J.-L. Bougeret, M.L. Kaiser, S. Krucker, D.E. Larson, et al., GRL 26/11, 1573 (1999)

A. Balogh, R.G. Marsden, E.J. Smith (eds.), The Heliosphere Near Solar Minimum - The Ulysses Perspective (Springer-Praxis Books in Astrophysics and Astronomy, Praxis, Chichester, UK, and Springer, New York, 2001)

T.S. Bastian, D.E. Gary, JGR 102/A7, 14,031 (1997)

T.S. Bastian, M. Pick, A. Kerdraon, D. Maia, A. Vourlidas, ApJ 558, L65 (2001)

D.A. Batchelor, Solar Phys. 155, 57 (1994)

R. Berton, T. Sakurai, Solar Phys. 96, 93 (1985)

D.E. Billings, A Guide to the Solar Corona (Academic Press, New York, 1966)

V. Bothmer, R. Schwenn, Ann. Geophysicae 16, 1 (1998)

V. Bothmer, Sources of Magnetic Helicity Over the Solar Cycle, Proc. ISCS 2003 Symposium Solar Variability as an Input to the Earth's Environment (Tatranska Lomnica, Slovakia, 23-28 June 2003), ESA SP-535, p. 419

I.H. Cairns, M.L. Kaiser, in The Review of Radio Science 1999-2002, ed. by W.R. Stone (IEEE Press, 2002), p. 749

H.V. Cane, N.R. Sheeley Jr., R.A. Howard, JGR 92, 9869 (1987)

H.V. Cane, W.C. Erickson, ApJ 623, 1180 (2005)

C. Caroubalos, J.-L. Steinberg, A\&A 32, 245 (1974) 
C. Caroubalos, M. Poquerusse, J.-L. Steinberg, A\&A 32, 255 (1974)

J. Chen, R.A. Howard, G.E. Brueckner, R. Santoro, J. Krall, S.E. Paswaters, et al., ApJ 409, L191 (1997)

J. Chen, R.A. Santoro, J. Krall, R.A. Howard, R. Duffin, J.D. Moses, et al., ApJ 533, 481 (2000)

P.F. Chen, S.T. Wu, K. Shibata, C. Fang, ApJ 572, L99 (2002)

A. Ciaravella, J.C. Raymond, B.J. Thompson, A. Van Ballegooijen, L. Strachan, L. Gardner, et al., ApJ 529, 575 (2001)

S.R. Cranmer, Space Sci. Rev. 101/3, 229 (2002)

H. Cremades, V. Bothmer, A\&A 422, 307 (2004)

J.M. Davila, ApJ 423, 871 (1994)

J.M. Davila, D.M. Rust, V.J. Pizzo, C. Paulett, in Solar Terrestrial Relations Observatory (STEREO), ed. by D. M. Rust. SPIE Proc. Missions to the Sun, vol. 2804 (SPIE, 1996), p. 34

J.M. Davila, O. St. Cyr, Science Prospects for the STEREO Mission, in Proc. 34th COSPAR Scientific Assembly, The Second World Space Congress (Houston, Texas, Oct 10-19, 2002), p. 478

K.P. Dere, E. Landi, H.E. Mason, B.C. Monsignori-Fossi, P.R. Young, A\&A 125, 149 (1997)

K.P. Dere, E. Landi, P.R. Young, G. Del Zanna, ApJS 134, 331 (2001)

K.P. Dere, G.E. Brueckner, R.A. Howard, D.J. Michels, J.P. Delaboudiniere, ApJ 516, 465 (1999)

G.A. Dulk, J.L. Steinberg, A. Lecacheux, S. Hoang, R.J. MacDowall, A\&A 150, L28 (1985)

T.G. Forbes, JGR 105/A10, 23,153 (2000)

C. Foullon, in Proc. ISCS Symposium on Solar Variability ans an Input to the Earth's Environment, vol. 535, ed. by A. Wilson (ESA, ESTEC Noordwijk, The Netherlands, ESA-SP, 2003), p. 477

R.A. Frazin, ApJ 530, 1026 (2000)

R.A. Frazin, P. Janzen, ApJ 570, 408 (2002)

R.A. Frazin, F. Kamalabadi, M.A. Weber, ApJ 628, 1070 (2005)

R.A. Frazin, F. Kamalabadi, ApJ 628, 1061 (2005)

A. Gary, Solar Phys. 174, 241 (1997)

A. Gary, J.M. Davis, R. Moore, Solar Phys. 183, 45 (1998)

A. Gary, D. Alexander, Solar Phys. 186, 123 (1999)

A. Gary, R.L. Moore, ApJ 611, 545 (2004)

J. Giacalone, J.R. Jokipii, J. Kóta, ApJ 573, 845 (2002)

S.E. Gibson, B.C. Low, ApJ 493, 460 (1998)

S.E. Gibson, B.C. Low, JGR 105/A8, 18,187 (2000)

N. Gopalswamy, M.R. Kundu, Solar Phys. 143, 327 (1993)

N. Gopalswamy, S. Yashiro, M.L. Kaiser, R.A. Howard, and J.-L.H. Bougeret, ApJ 548, L91 (2001)

V.M. Grigoryev, Solar Phys. 148, 389 (1993)

C.P.T. Groth, D.L. DeZeeuw, T.I. Gombosi, K.G. Powell, JGR 105, 25,053 (2000)

B.V. Gudiksen, A. Nordlund, ApJ 572, L113 (2002)

B.V. Gudiksen, A. Nordlund, ApJ 618, 1020 (2005a)

B.V. Gudiksen, A. Nordlund, ApJ 618, 1031 (2005b)

D.A. Gurnett, M.M. Baumback, H. Rosenbauer, JGR 83, 616 (1978)

J.R. Hall, P. Sheth, E. DeJong, J. Lorre, P. Liewer, 3D Sun Loop Tracer: A Tool for Stereoscopy of Coronal Loops for NASA's STEREO Mission (American Geophysical Union, Fall Meeting 2004, abstract \#SH21B, 2004)

R.A. Howard, J.D. Moses, D.G. Socker, The SECCHI Consortium, Adv. Space Res. 29, 2017 (2002)

J.T. Hoeksema, Structure and evolution of the large scale solar and heliospheric magnetic fields, PhD Thesis (Stanford University, California, 1984)

H.S. Hudson, J.R. Lemen, O.C. St.Cyr, A.C. Sterling, D.F. Webb, GRL 25, 2481 (1998)

N.E. Hurlburt, P.C.H. Martens, G.L. Slater, S.M. Jaffey, in Solar Active Region Evolution: Comparing Models with Observations, ed. by K.S. Balasubramaniam, G.W. Simon, 14th Internat. Summer NSO Workshop, vol. 68 (Astronomical Society of the Pacific (ASP), San Francisco, ASP Conference Series, 1994), p. 30

N.E. Hurlburt, D. Alexander, A.M. Rucklidge, ApJ 577, 993 (2002)

S.S. Ipson, V.V. Zharkova, S. Zharkov, A.K. Benkhalil, J. Abourdarham, N. Fuller, Solar Phys. 228, 399 (2005)

B.V. Jackson, H.R. Froehling, A\&A 299, 885 (1995)

B.V. Jackson, P.P. Hick, Solar Phys. 211, 345 (2002)

B.V. Jackson, P.P. Hick, in Solar and Space Weather Radiophysics, ed. by D.E. Gary, C.O. Keller, Astrophysics and Space Science Library, vol. 314 (Kluwer Academic Publishers, Dordrecht, The Netherlands, 2004), p. 355

S.W. Kahler, in Coronal Mass Ejections, ed. by N. Crooker, J. Joselyn, J. Feynman, Proc. Chapman Conference, Geophysical Monograph Series, vol. 99 (American Geophysical Union (AGU), Washington, DC, 1997), p. 197

R. Kano, S. Tsuneta, ApJ 454, 934 (1995) 
B. Kliem, V.S. Titov, T. Török, A\&A 413, L23 (2004)

J.A. Klimchuk, in Space Weather, ed. by P. Song, H.J. Singer, G.L. Siscoe, American Geophysical Union, Geophysical Monograph Series, vol. 125, Proc. Chapman Conference on Space Weather, Clearwater, Florida, 2000 Mar 20-24 (AGU, Washington DC, 2001), p. 143

S.A. Knock, I.H. Cairns, P.A. Robinson, Z. Kuncic, JGR 106/A11, 25,041 (2001)

S.A. Knock, I.H. Cairns, P.A. Robinson, JGR 108/A10, SSH 2-1 CiteID 1361, DOI 10.1029/2003JA009960 (2003a)

S.A. Knock, I.H. Cairns, P.A. Robinson, Z. Kuncic, JGR 108/A3, SSH 6-1 CiteID 1126, DOI 10.1029/2002JA009508 (2003b)

S.A. Knock, I.H. Cairns, JGR 110/A1 (2005), CiteID A01101, DOI 10.1029/2004JA010452

M. Kojima, K.-I. Fujiki, M. Hirano, M. Tokumaru, T. Ohmi, K. Hakamada, in The Sun and the Heliosphere as an Integrated System, ed. by P. Poletto, S.T. Suess, Astrophysics and Space Science Library, vol. 317 (Kluwer Academic Publishers, Dordrecht, The Netherlands, 2004), p. 147

S. Koutchmy, M.M. Molodensky, Nature 360, 717 (1992)

H. Lamy, V. Pierrard, M. Maksimovic, J.F. Lemaire, JGR 108, 1047, pp. SSH 13-1 (2003), CiteID 1047, DOI 10.1029/2002JA009487

P.L. Lamy, E. Quemerais, A. Llebaria, M. Bout, R. Howard, R. Schwenn, et al., ESA-SP 404, 491 (1997)

E. Landi, M. Landini, K.P. Dere, P.R. Young, H.E. Mason, A\&AS 135, 339 (1999)

J.K. Lee, T.S. Newman, G.A. Gary, Oriented Connectivity-Based Method for Sgmenting Solar Loops, Proc. 17th Internat Conf., Pattern Recognition, vol. 4 (Journal, Elsevier, 2005), p. 315

M.A. Lee, ApJS 158, 38 (2005)

P.C. Liewer, J.M. Davis, E.M. DeJong, G.A. Gary, J.A. Klimchuk, R.P. Reinert, SPIE 3442, 53 (1998)

P.C. Liewer, E.M. DeJong, J.R. Hall, J. Lorre, EOS 81/48 (November 28, 2000 issue), p. F971

P.C. Liewer, J.R. Hall, M. DeJong, D.G. Socker, R.A. Howard, P.C. Crane, et al., JGR 106/A8, 15,903 (2001)

J. Lin, T.G. Forbes, JGR 105/A2, 2375 (2000)

J. Lin, A.A. van Ballegooijen, ApJ 629, 582 (2005)

J.A. Linker, G. VanHoven, D.D. Schnack, GRL 17/12, 2282 (1990)

J.A. Linker, Z. Miki, ApJ 438, L45 (1995)

J.A. Linker, Z. Mikić, D.A. Biesecker, R.J. Forsyth, S.E. Gibson, D. Biesecker, et al., JGR 104/A5, 9809 (1999)

J.A. Linker, R. Lionello, Z. Mikić, T. Amari, JGR 106/A11, 25,165 (2001)

R. Lionello, M. Velli, G. Einaudi, Z. Mikić, ApJ 494, 840 (1998)

A. Llebaria, P.L. Lamy, S. Koutchmy, ESA-SP 446, 441 (1999)

R.E. Loughhead, J.L. Wang, G. Blows, ApJ 274, 883 (1983)

B.C. Low, Solar Phys. 167, 217 (1996)

N. Lugaz, W.B. Manchester IV, T.I. Gombosi, ApJ 627, 1019 (2005)

J.G. Luhmann, S.C. Solomon, J.A. Linker, J.G. Lyon, Z. Mikić, D. Odstrčil, et al., J. Atmos. Solar-Terr. Phys. 66/15-16, 1243 (2004)

D.J. McLean, N.R. Labrum (eds.), Solar Radiophysics (Cambridge University Press, Cambridge, 1985)

W. Manchester IV, JGR 108/A4, 1162, SSH 10-1 (2003), DOI 10.1029/2002JA009252

W. Manchester IV, T.I. Gombosi, I. Roussev, D.L. DeZeeuw, I.V. Sokolov, K.G. Powell, et al., JGR 109/A1, A01102 (2004a)

W. Manchester IV, T. Gombosi, D. DeZeeuw, Y. Fan, ApJ 610, 588 (2004b)

Z. Mikić, J.A. Linker, ApJ 430, 898 (1994)

Z. Mikić, J.A. Linker, D.D. Schnack, R. Lionello, A. Tarditi, Phys. Plasmas 6/No.5, 2217 (1999)

E.A. Milne, MNRAS 81, 361 (1921)

M. Minnaert, Zeitschrift fur Astrophysik 1, 209 (1930)

Y. Mok, Z. Mikić, R. Lionello, J.A. Linker, ApJ 621, 1098 (2005)

T.G. Moran, J.M. Davila, Science 305, 66 (2004)

J.T. Mueller, H. Maldonado, A.S. Driesman, Acta Astronautica 53, 729 (2003)

N. Neckel, D. Labs, Solar Phys. 153, 91 (1994)

G.J. Nelson, D.B. Melrose, in Solar Radiophysics, ed. by D.J. McLean, N.R. Labrum (Cambridge University Press, Cambridge, 1985), p. 333

C.K. Ng, D.V. Reames, A.J. Tylka, GRL 26, 2145 (1999)

C.K. Ng, D.V. Reames, A.J. Tylka, ApJ 591, 461 (2003)

M. Neugebauer, in The Heliosphere Near Solar Minimum - The Ulysses Perspective, ed. by A. Balogh, R.G. Marsden, E.J. Smith (Springer-Praxis Books in Astrophysics and Astronomy, 2001), p. 43

D. Odstrčil, JGR 99/A9, 17,653 (1994)

D. Odstrčil, M. Dryer, Z. Smith, JGR 101, 19,973 (1996)

D. Odstrčil, V.J. Pizzo, JGR 104/A12, 28,225 (1999a) 
D. Odstrčil, V.J. Pizzo, JGR 104/A1, 483 (1999b)

D. Odstrčil, V.J. Pizzo, JGR 104/A1, 493 (1999c)

D. Odstrčil, M. Karlický, A\&A 359, 766 (2000)

D. Odstrčil, J.A. Linker, R. Lionello, Z. Mikić, P. Riley, V.J. Pizzo, et al., JGR 107/A12, SSH 14-1 (2002), CiteID 1493, DOI 10.1029/2002JA009334

D. Odstrčil, Adv. Space Res. 32/4, 497 (2003)

D. Odstrčil, P. Riley, X.P. Zhao, JGR, 109/A2 (2004a), CideID A02116, DOI 10.1029/2003JA010135

D. Odstrčil, V.J. Pizzo, J.A. Linker, P. Riley, R. Lionello, Z. Mikić, J. Atmos. Solar-Terr. Phys. 66, 1311 (2004b)

D. Odstrčil, V.J. Pizzo, C.N. Arge, JGR (Space Phys.) 110, 2106 (2005), CiteID A02106, DOI 10.1029/2004JA010745

M. Pick, D. Maia, A. Kerdraon, R.A. Howard, G.E. Brueckner, D.J. Michels, et al., Solar. Phys. 181, 455 (1998)

V.J. Pizzo (ed.), The Findings of the SPINS Science Workshop, SEL Workshop Report January 1994, SPINS: Special Perscpectives Investigations, NOAA/SEL, Boulder, Colorado, on November 3-5, (1993)

V.J. Pizzo, D.A. Biesecker, GRL 31, 21,802 (2004)

O.V. Podladchikova, D. Berghmans, Solar Phys. 228, 265 (2005)

F. Portier-Fozzani, B. Inhester, Space Sci. Rev. 97, (1/4), 51 (2001)

F. Portier-Fozzani, B. Inhester, in Magnetic Coupling of the Solar Atmosphere, Proc. Euroconference and IAU Colloquium 188, vol. 505 (ESA, ESTEC Noordwijk, The Netherlands, ESA-SP, 2002), p. 545

K.G. Powell, P.L. Roe, T.J. Linde, T.I. Gombosi, D.L. deZeeuw, J. Comp. Phys. 154, 284 (1999)

R.C. Puetter, Int. J. Image Sys. Tech. 6, 314 (1995)

R.C. Puetter, SPIE 2827, 12 (1996)

R.C. Puetter, in Instrumentation for Large Telescopes, ed. by J.M. Rodriguez Espinosa, A. Herrero, F. Sanchez (Cambridge University Press, New York, 1997), p. 75

R.C. Puetter, A. Yahil, in Astronomical Data Analysis Software and Systems VIII, ASP Conference Series, vol. 172, ed. by D.M. Mehringer, R.L. Plante, D.A. Roberts (1999), p. 307

E. Qué merais, P. Lamy, A\&A 393295 (2002)

D.V. Reames, in Coronal Mass Ejections, ed. by N. Crooker, J. Joselyn, J. Feynman, Proc. Chapman Conference, Geophysical Monograph Series, vol. 99 (American Geophysical Union (AGU), Washington, DC, 1997), p. 217

M.J. Reiner, R.G. Stone, Solar Phys. 100, 397 (1986)

M.J. Reiner, R.G. Stone, A\&A 206, 316 (1988)

M.J. Reiner, R.G. Stone, A\&A 217, 251 (1989)

M.J. Reiner, M.L. Kaiser, J. Fainberg, J.-L. Bougeret, R.G. Stone, GRL 25/14, 2493 (1998a)

M.J. Reiner, J. Fainberg, M.L. Kaiser, R.G. Stone, JGR 103, 1923 (1998b)

M.J. Reiner, M.L. Kaiser, JGR 104/A8, 16,979 (1999)

P. Riley, J.A. Linker, Z. Mikić, JGR 106/A8, 15,889 (2001a)

P. Riley, J.A. Linker, Z. Mikić, R. Lionello, in Space Weather, Geophysical Monograph Series, vol. 125, ed. by P. Song, H.J. Singer, G.L. Siscoe (2001b), p. 159

P. Riley, J.A. Linker, Z. Mikić, JGR 107/A7 (2002a), DOI 10.1029/2001JA000299

P. Riley, J.A. Linker, Z. Mikić, D. Odstrčil, V.J. Pizzo, D.F. Webb, ApJ 578, 972 (2002b)

P. Riley, Z. Mikić, J.A. Linker, Ann. Geophys. J. 21, 1347 (2003a)

P. Riley, J.A. Linker, Z. Mikić, D. Odstrčil, T.H. Zurbuchen, D. Lario, et al., JGR 108/A7, SSH 2-1 (2003b), CiteID 1272, DOI 10.1029/2002JA009760

P. Riley, J.A. Linker, R. Lionello, Z. Mikić, D. Odstrčil, M.A. Hidalgo, Q. Hu, R.P. Lepping, B.J. Lynch, A. Rees, J. Atmos. Solar-Terr. Phys. 66, 1321 (2004)

P. Riley, N.U. Crooker, ApJ 600, 1035 (2004)

P.A. Robinson, I.H. Cairns, Solar Phys. 181, 363 (1998a)

P.A. Robinson, I.H. Cairns, Solar Phys. 181, 395 (1998b)

P.A. Robinson, I.H. Cairns, Solar Phys. 181, 429 (1998c)

E. Robbrecht, D. Berghmans, A\&A 425, 1097 (2004)

R. Rosner, W.H. Tucker, G.S. Vaiana, ApJ 220, 643 (1978)

I.I. Roussev, T.I. Gombosi, I.V. Sokolov, M. Velli, W. Manchester, D.L. DeZeeuw, et al., ApJ 595, L57 (2003a)

I.I. Roussev, T.G. Forbes, T.I. Gombosi, I.V. Sokolov, D.L. DeZeeuw, J. Birn, ApJ 588, L45 (2003b)

I.I. Roussev, I.V. Sokolov, T.G. Forbes, T.I. Gombosi, M.A. Lee, J.I. Sakai, ApJ 605, L73 (2004)

I.I. Roussev, I.V. Sokolov, in Solar Eruptions and Energetic Particles, Chapman Conf., Turku, Finland, 2-6 Aug 2004, Geophysical Monograph Series, ed. by N. Gopalswamy, R. Mewaldt, J. Torsti (AGU, Washington, DC, 2005, in press)

D.M. Rust, A. Kumar, ApJ 464, L199 (1996) 
D.M. Rust, et al. (eds.), The sun and heliosphere in three dimensions, Report of the NASA Science Definition Team for the STEREO Mission (edited and published at John Hopkins Univ. Applied Physics Lab., Laurel, Maryland, 1997 Dec 1)

D.M. Rust, B. LaBonte, ApJ 622, L69 (2005)

K.H. Schatten, J.M. Wilcox, N.F. Ness, Solar Phys. 6, 442 (1969)

W.K.H. Schmidt, V. Bothmer, Adv. Space Res. 17, 369 (1996)

C.J. Schrijver, M.L. DeRosa, Solar Phys. 212, 165 (2003)

C.J. Schrijver, A.W. Sandman, M.J. Aschwanden, M.L. DeRosa, ApJ 615, 512 (2004)

R. Schwenn, E. Marsch (eds.), Physics of the Inner Heliosphere. I. Large-Scale Phenomena (Springer-Verlag, Berlin, 1991a), P. 282

R. Schwenn, E. Marsch, (eds.), Physics of the Inner Heliosphere. II. Particles, Waves and Yurbulence, (SpringerVerlag Berlin, 1996) P. 352

S. Serio, G. Peres, G.S. Vaiana, L. Golub, R. Rosner, ApJ 243, 288 (1981)

D.G. Socker, et al., SPIE 2804, 50 (1996)

D.G. Socker, SPIE 3442, 44 (1998)

D.G. Socker, et al:: SPIE 4139, 284 (2000)

I.V. Sokolov, E.V. Timofeev, J.I. Sakai, K. Takayama, J. Comp. Phys. 181, 354 (2002)

I.V. Sokolov, I.I. Roussev, T.I. Gombosi, M.A. Lee, J. Kóta, T.G. Forbes, et al., ApJ 616, L171 (2004)

R.S. Steinolfson, A.J. Hundhausen, JGR 93, 14,269 (1988)

R.T. Stewart, Solar Phys. 94, 379 (1984)

B.J. Thompson, J.B. Gurman, W.M. Neupert, J.S. Newmark, J.P. Delaboudiniere, O.C. St.Cyr, et al., ApJ 517, L151 (1999)

V.S. Titov, P. Démoulin, A\&A 351, 707 (1999)

T. Török, B. Kliem, A\&A 406, 1043 (2003)

T. Török, B. Kliem, V.S. Titov, A\&A 413, L27 (2003)

T. Török, B. Kliem, in Coronal Heating, SOHO-15 Workshop, vol. 575 (ESA, ESTEC Noordwijk, The Netherlands, ESA-SP, 2004), p. 56

G. Toth, Astrophys. Lett. Comm. 34, 245 (1996)

G. Toth, D. Odstrčil, J. Comput. Phys. 182, 82 (1996)

D. Tripathi, V. Bothmer, H. Cremades, A\&A 422, 337 (2004)

A.J. Tylka, D.V. Reames, C.K. Ng, GRL 26/14, 2141 (1999)

A.J. Tylka, C.M.S. Cohen, W.F. Dietrich, M.A. Lee, C.G. Maclennan, R.A. Mewaldt, et al., ApJ 625, 474 (2005)

H.C. Van de Hulst, Bull. Astron. Inst. Neth. 11, 135 (1950)

A.A. Vedenov, V.A. Koutvitsky, S. Koutchmy, M.M. Molodensky, V.N. Oraevsky, Astronomy Reports 44/2, 112 (2000)

A. Vourlidas, P. Subramanian, K.P. Dere, R.A. Howard, ApJ 534, 456 (2000)

A. Vourlidas, R.A. Howard, ApJ 642, 1216 (2006)

Y.M. Wang, N.R. Sheeley Jr., ApJ 392, 310 (1992)

Y.M. Wang, ApJ 543, L89 (2000)

A. Warmuth, G. Mann, A\&A 435, 1123 (2005)

D.F. Webb, J. Burkepile, T.G. Forbes, P. Riley, JGR 108/A12, SSH 6-1 (2003), CiteID 1440, DOI 10.1029/2003JA009923

T. Wiegelmann, T. Neukirch, Solar Phys. 208, 233 (2002)

T. Wiegelmann, B. Inhester, Solar Phys. 214, 287 (2003)

T. Wiegelmann, A. Lagg, S.K. Solanki, B. Inhester, J. Woch, A\&A 433, 701 (2005)

T. Wiegelmann, B. Inhester, A. Lagg, S. Solanki, Solar Phys. 228, 67 (2005)

T. Wiegelmann, B. Inhester, Solar Phys. (2006, in press)

J.P. Wild, S.F. Smerd, A.A. Weiss, Annu. Rev. Astron. Astrophys. 1, 291 (1963)

A.R. Winebarger, H. Warren, A. VanBallegooijen, E. DeLuca, L. Golub, ApJ 567, L89 (2002)

B.E. Wood, M. Karovska, J. Chen, G.E. Brueckner, J.W. Cook, R.A. Howard, ApJ 512, 484 (1999)

S.T. Wu, H. Zheng, S. Wang, B.J. Thompson, S.P. Plunkett, X.P. Zhao, et al., JGR 106, 25,089 (2001)

P.R. Young, E. Landi, R.J. Thomas, A\&A 329, 391 (1998)

G.P. Zank, G. Li, Particle acceleration at CME-driven shocks: why the injection problem is important, in Proc. 35th COSPAR Scientific Assembly, 18-25 July 2004, Paris, France, p. 295

Ya.B. Zeldovich, Yu.P. Raizer, Physics of Shock Waves and High-Temperature Hydrodynamic Phenomena, ed. by W.D. Hayes, R.F. Probstein (Mineola, Dover, 2002)

M. Zhang, B.C. Low, Annu. Rev. Astron. Astrophys. 43, 103 (2005)

V.V. Zharkova, V. Schetinin, Solar Phys. 228, 137 (2005) 
S. Zidowitz, B. Inhester, A. Epple, in Solar Wind Eight, ed. by D. Winterhalter, J.T. Gosling, S.R. Habbal, W.S. Kurth, M. Neugebauer, Internat, Solar Wind Conference, American Institute of Physics Conference Proceedings AIP-CP, vol. 382 (AIP Press, New York, 1996), p. 165

S. Zidowitz, in, The Corona and Solar Wind Near Minimum Activity, ed. by A. Wilson, Proc. 5th SoHO Workshop, vol. 404 (European Space Agency, ESTEC Noordwijk, The Netherlands, ESA-SP, 1997), p. 757

S. Zidowitz, JGR 104/A5, 9727 (1999) 